\title{
LIMIT CYCLES OF CUBIC POLYNOMIAL DIFFERENTIAL SYSTEMS WITH RATIONAL FIRST INTEGRALS OF DEGREE 2
}

\author{
JAUME LLIBRE ${ }^{1}$, BRUNO D. LOPES ${ }^{1,2}$ AND JAIME R. DE MORAES ${ }^{2}$
}

\begin{abstract}
The main goal of this paper is to study the maximum number of limit cycles that bifurcate from the period annulus of the cubic centers that have a rational first integral of degree 2 when they are perturbed inside the class of all cubic polynomial differential systems using the averaging theory. The computations of this work have been made with Mathematica and Maple.
\end{abstract}

\section{Introduction and Statement of the Main Results}

One of the main problems in the qualitative theory of real planar differential systems is the determination of their limit cycles. There are several methods for computing the number of limit cycles bifurcating from the periodic orbits of a center $[1,2]$. Most of the methods are based on the Poincaré return map, the Poincaré-Melnikov integrals (see for instance $[8,9]$ ), the Abelian integrals, and the averaging theory of first order. In fact in the plane the last three methods are essentially equivalent. The first two methods only give the number of the periodic orbits of the unperturbed system that become limit cycles when the system is perturbed. The averaging method and method presented in [5] using the inverse integrating factor also give the shape of the bifurcated limit cycles. For a general overview of some of these mentioned tools see the book [7].

The study of the number of limit cycles of a polynomial differential system is mainly motivated by the 16-th Hilbert problem, which together with the Riemann conjecture are the two problems of the famous list of 23 problems of Hilbert which remains open. See for more details [10] and [16].

The problem of studying the bifurcation of limit cycles from the periodic orbits of a center of a polynomial differential system of degree 2 when this system is perturbed inside the class of all polynomial differential systems of degree 2 has been studied intensively during these last 20 years, see for instance the books [4] and [18], and the hundreds of references quoted there, and in particular the references $[6,13,19]$. There are few works trying to study this problem for cubic polynomial differential systems. Our objetive

2010 Mathematics Subject Classification. 34A36, 34C07, 34C25, 34C29.

Key words and phrases. polynomial vector fields, limit cycles, isochronous centers, periodic orbits, averaging method. 
will be to study this problem for the cubic polynomial differential systems having a rational first integral of degree 2 .

The classification of all cubic polynomial differential systems having a center at the origin and a rational first integral of degree 2 can be found in [14]. Now we summarize this classification in six families of cubic polynomial differential systems that we denote by $P_{k}$ for $k=1,2, \ldots, 6$.

The class $P_{1}$ is represented by system

$$
\begin{aligned}
& \dot{x}=2 y\left(\alpha^{2}+\beta+2 \alpha x+x^{2}\right), \\
& \dot{y}=-2\left(\left(\alpha^{2}+\beta\right) x+\alpha x^{2}-\alpha y^{2}-x y^{2}\right),
\end{aligned}
$$

with $\beta<0$ and $\alpha^{2}+\beta \neq 0$.

The class $P_{2}$ is obtained translating the other center of $P_{1}$ to origin. It is described by system

$$
\begin{aligned}
& \dot{x}=2 \alpha^{-2} y\left(\beta^{2}-2 \alpha \beta x+\alpha^{2}\left(\beta+x^{2}\right)\right), \\
& \dot{y}=-2\left(\alpha^{2} x-\alpha x^{2}-\alpha^{-2} \beta y^{2}+x\left(\beta+y^{2}\right)\right) .
\end{aligned}
$$

The class $P_{3}$ is given by system

$$
\begin{aligned}
& \dot{x}=x\left(1+\alpha^{2}+2 x+x^{2}-y^{2}\right), \\
& \dot{y}=y\left(-1-\alpha^{2}-2 y+x^{2}-y^{2}\right),
\end{aligned}
$$

with $\alpha \neq 0$.

The class $P_{4}$ is represented by system

$$
\dot{x}=y\left(x^{2}+\alpha\right), \quad \dot{y}=x\left(y^{2}+\beta\right),
$$

with $\alpha \beta<0$.

The class $P_{5}$ is provided by system

$$
\begin{aligned}
& \dot{x}=x\left(\beta^{2}+d^{2}+2(\beta+\gamma d) x+\left(\gamma^{2}+1\right) x^{2}-y^{2}\right), \\
& \dot{y}=y\left(-\beta^{2}-d^{2}-2 d y+\left(\gamma^{2}+1\right) x^{2}-y^{2}\right),
\end{aligned}
$$

with $d=0$ or $d=1$. If $d=0$ then $\beta \gamma \neq 0$ and if $d=1$ then $\beta(\beta \gamma-1) \neq 0$.

Finally the class $P_{6}$ is obtained taking $\beta=0$ in $P_{1}$. Thus, we get the system

$$
\begin{aligned}
& \dot{x}=2 y(x+\alpha)^{2}, \\
& \dot{y}=-2(x+\alpha)\left(\alpha x-y^{2}\right),
\end{aligned}
$$

with $\alpha \neq 0$.

In [12] the authors studied the cubic polynomial differential systems having a rational first integral of degree 2 whose phase portraits correspond to the phase portraits $P_{1}, P_{3}$ and $P_{4}$ of Figure 1 . These systems were denoted in [12] by (A), (B) and (C). They also proved that all the centers of these systems are reversible and isochronous, see [12, p. 314]. Their main result provides upper bounds $\mathcal{M}$ for the maximum number of limit cycles of systems $P_{1}, P_{3}$ and $P_{4}$ when they are perturbed inside the class of all polynomial differential systems of degree 3 using the Abelian integrals, see the 
column "Upper bound" in Table 1. Here we study the maximum number of limit cycles $\mathcal{N}$ that bifurcate from the period annulus of the cubic reversible centers $P_{1}, P_{2}, P_{4}$ and $P_{6}$ when they are perturbed inside the class of all cubic polynomial differential systems using the averaging theory of first order and the maximum number of infinitesimal limit cycles $\mathcal{I}$ for the centers $P_{3}$ and $P_{5}$ by using the averaging theory of fifth order.

A natural question is: How many periodic solutions surrounding the origin persists as limit cycles when we perturb the systems $P_{1}-P_{6}$ inside the class of all cubic polynomial differential systems?

More specifically consider the following systems

$$
\begin{array}{ccc}
\dot{x}= & 2 y\left(\alpha^{2}+\beta+2 \alpha x+x^{2}\right)+\varepsilon p(x, y), \\
\dot{y}= & -2\left(\left(\alpha^{2}+\beta\right) x+\alpha x^{2}-\alpha y^{2}-x y^{2}\right)+\varepsilon q(x, y), \\
\dot{x}= & 2 \alpha^{-2} y\left(\beta^{2}-2 \alpha \beta x+\alpha^{2}\left(\beta+x^{2}\right)\right)+\varepsilon p(x, y), \\
\dot{y}= & -2\left(\alpha^{2} x-\alpha x^{2}-\alpha^{-2} \beta y^{2}+x\left(\beta+y^{2}\right)\right)+\varepsilon q(x, y), \\
\dot{x}= & x\left(\left(1+\alpha^{2}\right)+2 x+x^{2}-y^{2}\right)+\varepsilon p(x, y), \\
\dot{y}= & y\left(-\left(1+\alpha^{2}\right)-2 y+x^{2}-y^{2}\right)+\varepsilon q(x, y), \\
\dot{x}= & y\left(x^{2}+\alpha\right)+\varepsilon p(x, y), \quad \dot{y}=x\left(y^{2}+\beta\right)+\varepsilon q(x, y), \\
\dot{x}= & x\left(\beta^{2}+d^{2}+2(\beta+\gamma d) x+\left(\gamma^{2}+1\right) x^{2}-y^{2}\right)+\varepsilon p(x, y), \\
\dot{y}= & y\left(-\beta^{2}-d^{2}-2 d y+\left(\gamma^{2}+1\right) x^{2}-y^{2}\right)+\varepsilon q(x, y), \\
& \\
\dot{x}= & 2 y(x+\alpha)^{2}+\varepsilon p(x, y), \\
\dot{y}= & -2(x+\alpha)\left(\alpha x-y^{2}\right)+\varepsilon q(x, y),
\end{array}
$$

where

$p(x, y)=a_{1} x+a_{2} y+a_{3} x^{2}+a_{4} x y+a_{5} y^{2}+a_{6} x^{3}+a_{7} x^{2} y+a_{8} x y^{2}+a_{9} y^{3}$, $q(x, y)=b_{1} x+b_{2} y+b_{3} x^{2}+b_{4} x y+b_{5} y^{2}+b_{6} x^{3}+b_{7} x^{2} y+b_{8} x y^{2}+b_{9} y^{3}$,

of course, the parameters of the unperturbed system (1)-(6) (i.e. of the systems $\left.P_{1}-P_{6}\right)$ must satisfy conditions stated in systems $P_{1}-P_{6}$.

We compute the number $\mathcal{N}$ (see Table 1 ) for the classes $P_{1}, P_{2}, P_{4}$ and $P_{6}$. The Section 5 we explain why we cannot compute the number $\mathcal{N}$ for the classes $P_{3}$ and $P_{5}$. In Section 6 we calculate the maximum number of infinitesimal limit cycles $\mathcal{I}$ for the classes $P_{3}$ and $P_{5}$ when we perturb the classes inside the class of all cubic polynomial differential systems up to fifth order by using the averaging theory of fifth order. In Section 7 we give an example of a subfamily of the class $P_{5}$ that has at most 3 limit cycles by using the averaging theory of seventh order. In Appendix A we give explicitly two expressions used to find 3 limit cycles for the class $P_{5}$.

In what follows we present our main results. 

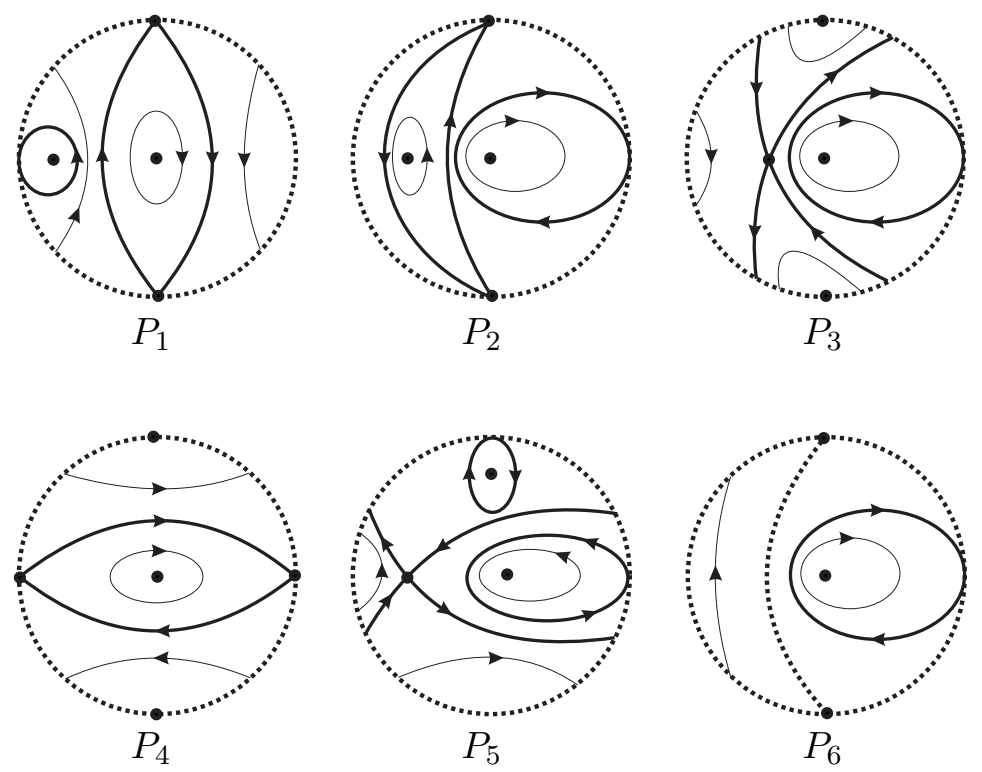

FIgURE 1. Phase portraits in the Poincaré disc of the cubic polynomial vector fields with a center at the origin and a rational first integral of degree 2 .

\begin{tabular}{|c|c|l|c|}
\hline Phase portraits & $\mathcal{M}$ & $\mathcal{N}$ & $\mathcal{I}$ \\
\hline$P_{1}$ & 4 & 3 & - \\
$P_{2}$ & 4 & 3 if $\left|\alpha^{2}+\beta\right| \geq 1$ & - \\
$P_{3}$ & 5 & $?$ & 2 \\
$P_{4}$ & 4 & 3 & - \\
$P_{5}$ & - & $?$ & 2 \\
$P_{6}$ & - & 3 & - \\
\hline
\end{tabular}

TABLE 1 . The second column presents the upper bounds $\mathcal{M}$ provided in [12], and the third column presents the maximum number reached $\mathcal{N}$ of limit cycles bifurcating from the periodic orbits surrounding the systems $P_{k}$ when they are perturbed inside the class of all cubic polynomial differential systems using the averaging theory of first order. The fourth column presents the maximum number reached $\mathcal{I}$ of infinitesimal limit cycles bifurcating from origin for systems $P_{k}$ using the averaging theory of fifth order.

Theorem 1. When we perturb systems $P_{1}, P_{4}$ and $P_{6}$ inside the class of all polynomial differential systems of degree three, the maximum number of limit cycles that bifurcate using the averaging theory of first order is $\mathcal{N}=3$ and it is reached. 
Theorem 1 is proved in Section 3.

Theorem 2. When we perturb system $P_{2}$ inside the class of all polynomial differential systems of degree three, the maximum number of limit cycles that bifurcate using the averaging theory of first order is $\mathcal{N}=3$ when $\alpha^{2}+\beta \notin$ $(-1,0) \cup(0,1)$ and it is reached.

Theorem 2 is proved in Section 4.

Theorem 3. When we perturb system $P_{3}$ and $P_{5}$ inside the class of all polynomial differential systems of degree three, the maximum number of infinitesimal limit cycles that bifurcate using the averaging theory of fifth order is 2, and it is reached.

Theorem 3 is proved in Section 6.

The proofs of Theorem 1,2 and 3 have been used in an essential way algebraic manipulators Mathematica and Maple.

In Section 2 we summarizes some preliminary results that we shall need for proving our theorems.

\section{Preliminaries}

The following theorem is a version of the averaging theory for arbitrary order which provides periodic solutions of a periodic continuous differential system. See [15] for a proof.

Consider the differential equation

$$
\dot{x}=\sum_{i=1}^{k} \varepsilon^{i} F_{i}(t, x)+\varepsilon^{k+1} R(t, x, \varepsilon),
$$

where $F_{i}: \mathbb{R} \times D \rightarrow \mathbb{R}^{n}$, for $i=1,2, \ldots, k$ and $R: \mathbb{R} \times D \times\left(-\varepsilon_{0}, \varepsilon_{0}\right) \rightarrow \mathbb{R}^{n}$ are continuous functions and $T$-periodic in the variable $t$, with $D$ is an open subset of $\mathbb{R}^{n}$.

We introduce some notations. Let $L$ be a positive integer, let $x=$ $\left(x_{1}, . ., x_{n}\right) \in D, t \in \mathbb{R}$ and $y_{j}=\left(y_{j 1}, \ldots, y_{j n}\right) \in \mathbb{R}^{n}$ for $j=1, \ldots, L$. Given $F: \mathbb{R} \times D \rightarrow \mathbb{R}^{n}$ a sufficiently smooth function, for each $(t, x) \in \mathbb{R} \times D$ we denote by $\partial^{L} F(t, x)$ a symmetric $L$-multilinear map which is applied to a "product" of $L$ vectors of $\mathbb{R}^{n}$, which we denote as $\bigodot_{j=1}^{L} y_{j} \in \mathbb{R}^{n L}$. The $L$-multilinear map is defined by

$$
\partial^{L} F(t, x) \bigodot_{j=1}^{L} y_{j}=\sum_{i_{1}, \ldots, i_{L}=1}^{n} \frac{\partial^{L} F(t, x)}{\partial x_{i_{1}}, \ldots, \partial x_{i_{L}}} y_{1 i_{1}}, \ldots, y_{L i_{L}} .
$$

Now we define the Averaged Function $f_{i}: D \rightarrow \mathbb{R}^{n}$ of order $i$ as

$$
f_{i}(z)=\frac{y_{i}(T, z)}{i !}
$$


where $y_{i}: \mathbb{R} \times D \rightarrow \mathbb{R}^{n}$ are given by

$$
\begin{aligned}
y_{i}(t, z)= & i ! \int_{0}^{t}\left(F_{i}(s, \varphi(s, z))\right. \\
& \left.+\sum_{l=1}^{i} \sum_{S_{l}} \frac{1}{b_{1} ! b_{2} ! 2 ! b^{2} \ldots b_{l} ! ! !^{b_{l}}} \partial^{L} F_{i-l}(s, \varphi(s, z)) \bigodot_{j=1}^{l} y_{j}(s, z)^{b_{j}}\right) d s .
\end{aligned}
$$

and $S_{l}$ is the set of all $l$-tuples of non-negative integers $\left(b_{1}, b_{2}, \ldots, b_{l}\right)$ satisfying $b_{1}+2 b_{2}+\ldots+l b_{l}=l$ and $L=b_{1}+b_{2}+\ldots+b_{l}$. For our purpose we give explicitly the functions $y_{i}$, for $i=1, \ldots, 5$. Thus we have

$$
\begin{aligned}
& y_{1}(t, z)= \int_{0}^{t} F_{1}(s, z) d s, \\
& y_{2}(t, z)= \int_{0}^{t}\left(2 F_{2}(s, z)+2 \frac{\partial F_{1}}{\partial x}(s, z) y_{1}(s, z)\right) d s \\
& y_{3}(t, z)= \int_{0}^{t}\left(6 F_{3}(s, z)+6 \frac{\partial F_{2}}{\partial x}(s, z) y_{1}(t, z)\right. \\
&\left.+3 \frac{\partial^{2} F_{1}}{\partial x^{2}}(s, z) y_{1}(s, z)^{2}+3 \frac{\partial F_{1}}{\partial x}(s, z) y_{2}(s, z)\right) d s \\
& y_{4}(t, z)= \int_{0}^{t}\left(24 F_{4}(s, z)+24 \frac{\partial F_{3}}{\partial x}(s, z) y_{1}(s, z)\right. \\
&+12 \frac{\partial^{2} F_{2}}{\partial x^{2}}(s, z) y_{1}(s, z)^{2}+12 \frac{\partial F_{2}}{\partial x}(s, z) y_{2}(s, z) \\
&+12 \frac{\partial^{2} F_{1}}{\partial x^{2}}(s, z) y_{1}(s, z) \odot y_{2}(s, z) \\
&\left.+4 \frac{\partial^{3} F_{1}}{\partial x^{3}}(s, z) y_{1}(s, z)^{3}+4 \frac{\partial F_{1}}{\partial x}(s, z) y_{3}(s, z)\right) d s \\
& \int_{0}^{t}\left(120 F_{5}(s, z)+120 \frac{\partial F_{4}}{\partial x}(s, z) y_{1}(s, z)+60 \frac{\partial^{2} F_{3}}{\partial x^{2}}(s, z) y_{1}(s, z)^{2}\right. \\
&+60 \frac{\partial F_{3}}{\partial x}(s, z) y_{2}(s, z)+60 \frac{\partial^{2} F_{2}}{\partial x^{2}}(s, z) y_{1}(s, z) \odot y_{2}(s, z) \\
&+20 \frac{\partial^{3} F_{2}}{\partial x^{3}}(s, z) y_{1}(s, z)^{3}+20 \frac{\partial F_{2}}{\partial x}(s, z) y_{3}(s, z) \\
&+20 \frac{\partial^{2} F_{1}}{\partial x^{2}}(s, z) y_{1}(s, z) \odot y_{3}(s, z) \\
&+15 \frac{\partial^{2} F_{1}}{\partial x^{2}}(s, z) y_{2}(s, z)^{2}+30 \frac{\partial^{3} F_{1}}{\partial x^{3}}(s, z) y_{1}(s, z)^{2} \odot y_{2}(s, z) \\
&\left.y_{5}(t, z)+5 \frac{\partial F_{1}}{\partial x}(s, z) y_{4}(s, z)\right) d s, \\
&=
\end{aligned}
$$

In Appendix A we give explicitly the expressions of $y_{6}$ and $y_{7}$ which we use to find 3 limit cycles for the class $P_{5}$. 
Theorem 4. Consider the initial value problem (7) and the averaged function (8), and assume the following conditions.

(i) For each $t \in \mathbb{R}, F_{i}(t, \cdot) \in \mathcal{C}^{k-i}$, for $i=1,2, \ldots, k ; \partial^{k-i} F_{i}$ is locally Lipschitz in the second variable for $i=1,2, \ldots, k$; and $R$ is continuous and locally Lipschitz in the second variable.

(ii) Assume that $f_{i}=0$ for $i=1, \ldots, r-1$ and $f_{r} \neq 0$ with $r \in\{1,2, \ldots, k\}$. Moreover, suppose that for some $a \in D$ with $f_{r}(a)=0$, there exists a neighborhood $V \subset D$ of a such that $f_{r}(z) \neq 0$ for all $z \in \bar{V} \backslash\{a\}$ and that $d_{B}\left(f_{r}(z), V, a\right) \neq 0$.

Then for $|\varepsilon|>0$ sufficiently small, there exists a T-periodic solution $x(\cdot, \varepsilon)$ of equation (7) such that $x(0, \varepsilon) \rightarrow a$ as $\varepsilon \rightarrow 0$.

Consider a planar differential system

$$
\dot{x}=P(x, y), \quad \dot{y}=Q(x, y),
$$

where $P, Q: \mathbb{R}^{2} \rightarrow \mathbb{R}$ are continuous functions. Suppose that system (9) has a continuous family of ovals

$$
\left\{\Gamma_{h}\right\} \subset\left\{(x, y): H(x, y)=h, h_{1}<h<h_{2}\right\}
$$

where $H$ is a first integral of (9). Consider the following perturbations of system (9)

$$
\dot{x}=P(x, y)+\varepsilon p(x, y), \quad \dot{y}=Q(x, y)+\varepsilon q(x, y),
$$

where $p, q: \mathbb{R}^{2} \rightarrow \mathbb{R}$ are continuous functions.

The following theorem (see Theorem 5.2 of [3] for a proof) provides a way for transforming the perturbed system (10) in the standard form of the averaging theory given in Theorem 4.

Theorem 5. Consider system (9) and its first integral $H$. Assume that $x Q(x, y)-y P(x, y) \neq 0$ for all $(x, y)$ in the period annulus formed by the ovals $\left\{\Gamma_{h}\right\}$. Let $\rho:\left(\sqrt{h_{1}}, \sqrt{h_{2}}\right) \times[0,2 \pi) \rightarrow[0, \infty)$ be a continuous function such that

$$
H(\rho(R, \varphi) \cos \varphi, \rho(R, \varphi) \sin \varphi)=R^{2},
$$

for all $R \in\left(\sqrt{h_{1}}, \sqrt{h_{2}}\right)$ and all $\varphi \in[0,2 \pi)$. Then the differential equation which describes the dependence between the square root of the energy $R=\sqrt{h}$ and the angle $\varphi$ for system (10) is

$$
\frac{d R}{d \varphi}=\varepsilon \frac{\mu\left(x^{2}+y^{2}\right)(Q p-P q)}{2 R(Q x-P y)}+\mathcal{O}\left(\varepsilon^{2}\right),
$$

where $\mu=\mu(x, y)$ is the integrating factor of system (9) associated to the first integral $H, x=\rho(R, \varphi) \cos \varphi$ and $y=\rho(R, \varphi) \sin \varphi$.

We recall that $\mu$ is the integrating factor associated to the first integral $H$ of system (9) if the equalities

$$
\mu P=\frac{\partial H}{\partial y} \quad \text { and } \quad \mu Q=-\frac{\partial H}{\partial x},
$$


hold.

The real functions $\left(f_{0}, f_{1}, \ldots, f_{n}\right)$ defined on $I$ is an Extended Chebyshev system or ET-system on $I$, if and only if any nontrivial linear combination of these functions has at most $n$ zeros counting their multiplicities and this number is reached. The functions $\left(f_{0}, f_{1}, \ldots, f_{n}\right)$ is an Extended Complete Chebyshev system or an ECT-system on $I$ if and only if for any $k \in\{0,1, \ldots, n\},\left(f_{0}, f_{1}, \ldots, f_{k}\right)$ form an ET-system. For proving that $\left(f_{0}, f_{1}, \ldots, f_{k}\right)$ is an ECT-system on $I$ is sufficient and necessary to prove that $W\left(f_{0}, \ldots, f_{k}\right)(s) \neq 0$ on $I$ for $k \in\{0,1, \ldots, n\}$. Let $W\left(f_{0}, \ldots, f_{k}\right)(s)$ be the Wronskian of the functions $\left(f_{0}, \ldots, f_{k}\right)$ with respect to $s$. We recall that the definition of the Wronskian is

$$
W\left(f_{0}, \ldots, f_{k}\right)(s)=\left|\begin{array}{cccc}
f_{0}(s) & f_{1}(s) & \cdots & f_{k}(s) \\
f_{0}^{\prime}(s) & f_{1}^{\prime}(s) & \cdots & f_{k}^{\prime}(s) \\
\vdots & \vdots & \ddots & \vdots \\
f_{0}^{(k)}(s) & f_{1}^{(k)}(s) & \cdots & f_{k}^{(k)}(s)
\end{array}\right|
$$

See [11] for more details on ECT-system.

In sequel we prove Theorem 1.

For commodity in the proofs of Theorems 1 and 2 we denote $\dot{x}=P(x, y)$ and $\dot{y}=Q(x, y)$ in the systems which represents the classes $P_{k}$, for $k=1,2,4$ and 6 .

\section{Proof of Theorem 1}

3.1. Proof for the class $P_{1}$. We have that

$$
\mu(x, y)=\frac{1}{\left(\alpha^{2}+2 \alpha x+\beta+x^{2}\right)^{2}},
$$

is the integrating factor associated to the first integral

$$
H(x, y)=\frac{x^{2}+y^{2}}{(\alpha+x)^{2}+\beta}
$$

of the system $P_{1}$, because they satisfy $\mu P=H_{y}$ and $\mu Q=-H_{x}$. By solving implicitly the equation $H(\rho \cos \theta, \rho \sin \theta)=R^{2}$ we obtain the positive function $\rho:(0,1) \rightarrow \mathbb{R}$ given by

$$
\rho=-\frac{R\left(\sqrt{4\left(\alpha^{2}+\beta\right)-2 \beta R^{2}\left(2 \cos ^{2} \theta-1\right)-2 \beta R^{2}}+2 \alpha R \cos \theta\right)}{R^{2}\left(2 \cos ^{2} \theta-1\right)+R^{2}-2} .
$$

So after the change of variable described in Theorem 5, system (1) becomes

$$
\frac{d R}{d \varphi}=\varepsilon \sum_{i=1}^{11} \frac{A_{i}(\varphi, \alpha, \beta, a, b)}{Q_{1}(R, \varphi, \alpha, \beta)} R^{i}+\mathcal{O}\left(\varepsilon^{2}\right)
$$


where $a=\left(a_{1}, \ldots, a_{9}\right), b=\left(b_{1}, \ldots, b_{9}\right)$ and

$$
\begin{aligned}
& A_{1}=-a_{1}\left(\alpha^{2}+\beta\right)^{2} \cos ^{2} \theta-\left(\alpha^{2}+\beta\right)^{2}\left(a_{2}+b_{1}\right) \sin \theta \cos \theta \\
& -b_{2}\left(\alpha^{2}+\beta\right)^{2} \sin ^{2} \theta \text {, } \\
& A_{2}=\sqrt{\alpha^{2}-\beta R^{2} \cos ^{2} \theta+\beta}\left(\left(-\alpha^{2}-\beta\right) \cos ^{3} \theta\left(\alpha^{2} a_{3}+3 \alpha a_{1}+a_{3} \beta\right)\right. \\
& -\left(\alpha^{2}+\beta\right) \sin ^{2} \theta \cos \theta\left(\alpha^{2} a_{5}+\alpha^{2} b_{4}-\alpha a_{1}+4 \alpha b_{2}+a_{5} \beta+\beta b_{4}\right) \\
& -\left(\alpha^{2}+\beta\right) \sin \theta \cos ^{2} \theta\left(\alpha^{2} a_{4}+\alpha^{2} b_{3}+3 \alpha a_{2}+4 \alpha b_{1}+a_{4} \beta+\beta b_{3}\right) \\
& \left.-\left(\alpha^{2}+\beta\right) \sin ^{3} \theta\left(\alpha^{2} b_{5}-\alpha a_{2}+\beta b_{5}\right)\right), \\
& A_{3}=-\left(\alpha^{2}+\beta\right)^{2} \sin ^{4} \theta\left(\alpha^{2} b_{9}-\alpha a_{5}+\beta b_{9}\right)-\left(\alpha^{2}+\beta\right) \sin ^{2} \theta \cos ^{2} \theta\left(\alpha^{4} a_{8}\right. \\
& +\alpha^{4} b_{7}-\alpha^{3} a_{3}+4 \alpha^{3} a_{5}+5 \alpha^{3} b_{4}-4 \alpha^{2} a_{1}+2 \alpha^{2} a_{8} \beta+2 \alpha^{2} \beta b_{7} \\
& \left.+4 \alpha^{2} b_{2}-\alpha a_{3} \beta+4 \alpha a_{5} \beta+5 \alpha \beta b_{4}-a_{1} \beta+a_{8} \beta^{2}+\beta^{2} b_{7}-4 \beta b_{2}\right) \\
& +\left(-\alpha^{2}-\beta\right) \cos ^{4} \theta\left(\alpha^{4} a_{6}+4 \alpha^{3} a_{3}+2 \alpha^{2} a_{6} \beta+4 \alpha a_{3} \beta-5 a_{1} \beta\right. \\
& \left.+a_{6} \beta^{2}\right)-\left(\alpha^{2}+\beta\right) \sin \theta \cos ^{3} \theta\left(\alpha^{4} a_{7}+\alpha^{4} b_{6}+4 \alpha^{3} a_{4}+5 \alpha^{3} b_{3}\right. \\
& +2 \alpha^{2} a_{7} \beta+2 \alpha^{2} \beta b_{6}+4 \alpha^{2} b_{1}+4 \alpha a_{4} \beta+5 \alpha \beta b_{3}-5 a_{2} \beta+a_{7} \beta^{2} \\
& \left.+\beta^{2} b_{6}-4 \beta b_{1}\right)-\left(\alpha^{2}+\beta\right) \sin ^{3} \theta \cos \theta\left(\alpha^{4} a_{9}+\alpha^{4} b_{8}-\alpha^{3} a_{4}+5 \alpha^{3} b_{5}\right. \\
& \left.-4 \alpha^{2} a_{2}+2 \alpha^{2} a_{9} \beta+2 \alpha^{2} \beta b_{8}-\alpha a_{4} \beta+5 \alpha \beta b_{5}-a_{2} \beta+a_{9} \beta^{2}+\beta^{2} b_{8}\right) \text {, } \\
& A_{4}=\sqrt{\alpha^{2}-\beta R^{2} \cos ^{2} \theta+\beta}\left(\alpha a_{9}\left(\alpha^{2}+\beta\right)^{2} \sin ^{5} \theta+\left(\alpha^{2}\right.\right. \\
& +\beta) \sin ^{4} \theta \cos \theta\left(\alpha^{3} a_{8}-6 \alpha^{3} b_{9}+5 \alpha^{2} a_{5}+\alpha a_{8} \beta-6 \alpha \beta b_{9}+a_{5} \beta\right) \\
& +\cos ^{5} \theta\left(-5 \alpha^{5} a_{6}-4 \alpha^{4} a_{3}+8 \alpha^{3} a_{1}-10 \alpha^{3} a_{6} \beta+12 \alpha a_{1} \beta-5 \alpha a_{6} \beta^{2}\right. \\
& \left.+4 a_{3} \beta^{2}\right)+\sin ^{2} \theta \cos ^{3} \theta\left(\alpha^{5} a_{6}-5 \alpha^{5} a_{8}-6 \alpha^{5} b_{7}+5 \alpha^{4} a_{3}-4 \alpha^{4} a_{5}\right. \\
& -9 \alpha^{4} b_{4}+4 \alpha^{3} a_{1}+2 \alpha^{3} a_{6} \beta-10 \alpha^{3} a_{8} \beta-12 \alpha^{3} \beta b_{7}+4 \alpha^{3} b_{2} \\
& +6 \alpha^{2} a_{3} \beta-6 \alpha^{2} \beta b_{4}+\alpha a_{6} \beta^{2}-5 \alpha a_{8} \beta^{2}-6 \alpha \beta^{2} b_{7}+12 \alpha \beta b_{2}+a_{3} \beta^{2} \\
& \left.+4 a_{5} \beta^{2}+3 \beta^{2} b_{4}\right)+\sin ^{3} \theta \cos ^{2} \theta\left(\alpha^{5} a_{7}-5 \alpha^{5} a_{9}-6 \alpha^{5} b_{8}+5 \alpha^{4} a_{4}\right. \\
& -9 \alpha^{4} b_{5}+4 \alpha^{3} a_{2}+2 \alpha^{3} a_{7} \beta-10 \alpha^{3} a_{9} \beta-12 \alpha^{3} \beta b_{8}+6 \alpha^{2} a_{4} \beta \\
& \left.-6 \alpha^{2} \beta b_{5}+\alpha a_{7} \beta^{2}-5 \alpha a_{9} \beta^{2}-6 \alpha \beta^{2} b_{8}+a_{4} \beta^{2}+3 \beta^{2} b_{5}\right) \\
& +\sin \theta \cos ^{4} \theta\left(-5 \alpha^{5} a_{7}-6 \alpha^{5} b_{6}-4 \alpha^{4} a_{4}-9 \alpha^{4} b_{3}+8 \alpha^{3} a_{2}\right. \\
& -10 \alpha^{3} a_{7} \beta-12 \alpha^{3} \beta b_{6}+4 \alpha^{3} b_{1}-6 \alpha^{2} \beta b_{3}+12 \alpha a_{2} \beta-5 \alpha a_{7} \beta^{2} \\
& \left.\left.-6 \alpha \beta^{2} b_{6}+12 \alpha \beta b_{1}+4 a_{4} \beta^{2}+3 \beta^{2} b_{3}\right)\right) \text {, } \\
& A_{5}=a_{9}\left(\alpha^{2}+\beta\right)^{2}\left(6 \alpha^{2}+\beta\right) \sin ^{5} \theta \cos \theta+\left(\alpha^{2}+\beta\right) \sin ^{4} \theta \cos ^{2} \theta\left(6 \alpha^{4} a_{8}\right. \\
& \left.-15 \alpha^{4} b_{9}+9 \alpha^{3} a_{5}+7 \alpha^{2} a_{8} \beta-12 \alpha^{2} \beta b_{9}+\alpha a_{5} \beta+a_{8} \beta^{2}+3 \beta^{2} b_{9}\right) \\
& +\sin ^{2} \theta \cos ^{4} \theta\left(6 \alpha^{6} a_{6}-9 \alpha^{6} a_{8}-15 \alpha^{6} b_{7}+9 \alpha^{5} a_{3}+4 \alpha^{5} a_{5}-5 \alpha^{5} b_{4}\right. \\
& -4 \alpha^{4} a_{1}+13 \alpha^{4} a_{6} \beta-14 \alpha^{4} a_{8} \beta-27 \alpha^{4} \beta b_{7}+10 \alpha^{4} b_{2}+10 \alpha^{3} a_{3} \beta \\
& +20 \alpha^{3} a_{5} \beta+10 \alpha^{3} \beta b_{4}-12 \alpha^{2} a_{1} \beta+8 \alpha^{2} a_{6} \beta^{2}-\alpha^{2} a_{8} \beta^{2}-9 \alpha^{2} \beta^{2} b_{7}
\end{aligned}
$$




$$
\begin{aligned}
& +12 \alpha^{2} \beta b_{2}+\alpha a_{3} \beta^{2}+16 \alpha a_{5} \beta^{2}+15 \alpha \beta^{2} b_{4}-4 a_{1} \beta^{2}+a_{6} \beta^{3}+4 a_{8} \beta^{3} \\
& \left.+3 \beta^{3} b_{7}-6 \beta^{2} b_{2}\right)+\cos ^{6} \theta\left(-9 \alpha^{6} a_{6}+4 \alpha^{5} a_{3}+6 \alpha^{4} a_{1}-14 \alpha^{4} a_{6} \beta\right. \\
& \left.+20 \alpha^{3} a_{3} \beta-\alpha^{2} a_{6} \beta^{2}+16 \alpha a_{3} \beta^{2}-10 a_{1} \beta^{2}+4 a_{6} \beta^{3}\right) \\
& +\sin ^{3} \theta \cos ^{3} \theta\left(6 \alpha^{6} a_{7}-9 \alpha^{6} a_{9}-15 \alpha^{6} b_{8}+9 \alpha^{5} a_{4}-5 \alpha^{5} b_{5}-4 \alpha^{4} a_{2}\right. \\
& +13 \alpha^{4} a_{7} \beta-14 \alpha^{4} a_{9} \beta-27 \alpha^{4} \beta b_{8}+10 \alpha^{3} a_{4} \beta+10 \alpha^{3} \beta b_{5}-12 \alpha^{2} a_{2} \beta \\
& +8 \alpha^{2} a_{7} \beta^{2}-\alpha^{2} a_{9} \beta^{2}-9 \alpha^{2} \beta^{2} b_{8}+\alpha a_{4} \beta^{2}+15 \alpha \beta^{2} b_{5}-4 a_{2} \beta^{2}+a_{7} \beta^{3} \\
& \left.+4 a_{9} \beta^{3}+3 \beta^{3} b_{8}\right)+\sin \theta \cos ^{5} \theta\left(-9 \alpha^{6} a_{7}-15 \alpha^{6} b_{6}+4 \alpha^{5} a_{4}-5 \alpha^{5} b_{3}\right. \\
& +6 \alpha^{4} a_{2}-14 \alpha^{4} a_{7} \beta-27 \alpha^{4} \beta b_{6}+10 \alpha^{4} b_{1}+20 \alpha^{3} a_{4} \beta+10 \alpha^{3} \beta b_{3} \\
& -\alpha^{2} a_{7} \beta^{2}-9 \alpha^{2} \beta^{2} b_{6}+12 \alpha^{2} \beta b_{1}+16 \alpha a_{4} \beta^{2}+15 \alpha \beta^{2} b_{3}-10 a_{2} \beta^{2} \\
& \left.+4 a_{7} \beta^{3}+3 \beta^{3} b_{6}-6 \beta^{2} b_{1}\right), \\
& A_{6}=\sqrt{\alpha^{2}-\beta R^{2} \cos ^{2} \theta+\beta}\left(3 \alpha a_{9}\left(\alpha^{2}+\beta\right)\left(5 \alpha^{2}+\beta\right) \sin ^{5} \theta \cos ^{2} \theta\right. \\
& +\sin ^{2} \theta \cos ^{5} \theta\left(15 \alpha^{5} a_{6}-5 \alpha^{5} a_{8}-20 \alpha^{5} b_{7}+5 \alpha^{4} a_{3}+10 \alpha^{4} a_{5}\right. \\
& +5 \alpha^{4} b_{4}-10 \alpha^{3} a_{1}+18 \alpha^{3} a_{6} \beta+10 \alpha^{3} a_{8} \beta-8 \alpha^{3} \beta b_{7}+4 \alpha^{3} b_{2} \\
& -6 \alpha^{2} a_{3} \beta+12 \alpha^{2} a_{5} \beta+18 \alpha^{2} \beta b_{4}-6 \alpha a_{1} \beta+3 \alpha a_{6} \beta^{2}+15 \alpha a_{8} \beta^{2} \\
& \left.+12 \alpha \beta^{2} b_{7}-12 \alpha \beta b_{2}-3 a_{3} \beta^{2}-6 a_{5} \beta^{2}-3 \beta^{2} b_{4}\right)+\cos ^{7} \theta\left(-5 \alpha^{5} a_{6}\right. \\
& +10 \alpha^{4} a_{3}-6 \alpha^{3} a_{1}+10 \alpha^{3} a_{6} \beta+12 \alpha^{2} a_{3} \beta-18 \alpha a_{1} \beta+15 \alpha a_{6} \beta^{2} \\
& \left.-6 a_{3} \beta^{2}\right)+\sin ^{3} \theta \cos ^{4} \theta\left(15 \alpha^{5} a_{7}-5 \alpha^{5} a_{9}-20 \alpha^{5} b_{8}+5 \alpha^{4} a_{4}+5 \alpha^{4} b_{5}\right. \\
& -10 \alpha^{3} a_{2}+18 \alpha^{3} a_{7} \beta+10 \alpha^{3} a_{9} \beta-8 \alpha^{3} \beta b_{8}-6 \alpha^{2} a_{4} \beta+18 \alpha^{2} \beta b_{5} \\
& \left.-6 \alpha a_{2} \beta+3 \alpha a_{7} \beta^{2}+15 \alpha a_{9} \beta^{2}+12 \alpha \beta^{2} b_{8}-3 a_{4} \beta^{2}-3 \beta^{2} b_{5}\right) \\
& +\sin \theta \cos ^{6} \theta\left(-5 \alpha^{5} a_{7}-20 \alpha^{5} b_{6}+10 \alpha^{4} a_{4}+5 \alpha^{4} b_{3}-6 \alpha^{3} a_{2}\right. \\
& +10 \alpha^{3} a_{7} \beta-8 \alpha^{3} \beta b_{6}+4 \alpha^{3} b_{1}+12 \alpha^{2} a_{4} \beta+18 \alpha^{2} \beta b_{3}-18 \alpha a_{2} \beta \\
& \left.+15 \alpha a_{7} \beta^{2}+12 \alpha \beta^{2} b_{6}-12 \alpha \beta b_{1}-6 a_{4} \beta^{2}-3 \beta^{2} b_{3}\right) \\
& +\sin ^{4} \theta \cos ^{3} \theta\left(15 \alpha^{5} a_{8}-20 \alpha^{5} b_{9}+5 \alpha^{4} a_{5}+18 \alpha^{3} a_{8} \beta-8 \alpha^{3} \beta b_{9}\right. \\
& \left.\left.-6 \alpha^{2} a_{5} \beta+3 \alpha a_{8} \beta^{2}+12 \alpha \beta^{2} b_{9}-3 a_{5} \beta^{2}\right)\right), \\
& A_{7}=a_{9}\left(\alpha^{2}+\beta\right)\left(20 \alpha^{4}-3 \alpha^{2} \beta-3 \beta^{2}\right) \sin ^{5} \theta \cos ^{3} \theta+\sin ^{2} \theta \cos ^{6} \theta\left(20 \alpha^{6} a_{6}\right. \\
& +5 \alpha^{6} a_{8}-15 \alpha^{6} b_{7}-5 \alpha^{5} a_{3}+4 \alpha^{5} a_{5}+9 \alpha^{5} b_{4}-4 \alpha^{4} a_{1}+17 \alpha^{4} a_{6} \beta \\
& +32 \alpha^{4} a_{8} \beta+15 \alpha^{4} \beta b_{7}-4 \alpha^{4} b_{2}-22 \alpha^{3} a_{3} \beta-12 \alpha^{3} a_{5} \beta+10 \alpha^{3} \beta b_{4} \\
& +6 \alpha^{2} a_{1} \beta-6 \alpha^{2} a_{6} \beta^{2}+21 \alpha^{2} a_{8} \beta^{2}+27 \alpha^{2} \beta^{2} b_{7}-16 \alpha^{2} \beta b_{2}-9 \alpha a_{3} \beta^{2} \\
& \left.-24 \alpha a_{5} \beta^{2}-15 \alpha \beta^{2} b_{4}+6 a_{1} \beta^{2}-3 a_{6} \beta^{3}-6 a_{8} \beta^{3}-3 \beta^{3} b_{7}+4 \beta^{2} b_{2}\right) \\
& +\cos ^{8} \theta\left(5 \alpha^{6} a_{6}+4 \alpha^{5} a_{3}-8 \alpha^{4} a_{1}+32 \alpha^{4} a_{6} \beta-12 \alpha^{3} a_{3} \beta-10 \alpha^{2} a_{1} \beta\right. \\
& \left.+21 \alpha^{2} a_{6} \beta^{2}-24 \alpha a_{3} \beta^{2}+10 a_{1} \beta^{2}-6 a_{6} \beta^{3}\right)+\sin ^{3} \theta \cos ^{5} \theta\left(20 \alpha^{6} a_{7}\right. \\
& +5 \alpha^{6} a_{9}-15 \alpha^{6} b_{8}-5 \alpha^{5} a_{4}+9 \alpha^{5} b_{5}-4 \alpha^{4} a_{2}+17 \alpha^{4} a_{7} \beta+32 \alpha^{4} a_{9} \beta \\
& +15 \alpha^{4} \beta b_{8}-22 \alpha^{3} a_{4} \beta+10 \alpha^{3} \beta b_{5}+6 \alpha^{2} a_{2} \beta-6 \alpha^{2} a_{7} \beta^{2}+21 \alpha^{2} a_{9} \beta^{2} \\
& \left.+27 \alpha^{2} \beta^{2} b_{8}-9 \alpha a_{4} \beta^{2}-15 \alpha \beta^{2} b_{5}+6 a_{2} \beta^{2}-3 a_{7} \beta^{3}-6 a_{9} \beta^{3}-3 \beta^{3} b_{8}\right) \\
& +\sin \theta \cos ^{7} \theta\left(5 \alpha^{6} a_{7}-15 \alpha^{6} b_{6}+4 \alpha^{5} a_{4}+9 \alpha^{5} b_{3}-8 \alpha^{4} a_{2}+32 \alpha^{4} a_{7} \beta\right.
\end{aligned}
$$




$$
\begin{aligned}
& +15 \alpha^{4} \beta b_{6}-4 \alpha^{4} b_{1}-12 \alpha^{3} a_{4} \beta+10 \alpha^{3} \beta b_{3}-10 \alpha^{2} a_{2} \beta+21 \alpha^{2} a_{7} \beta^{2} \\
& +27 \alpha^{2} \beta^{2} b_{6}-16 \alpha^{2} \beta b_{1}-24 \alpha a_{4} \beta^{2}-15 \alpha \beta^{2} b_{3}+10 a_{2} \beta^{2}-6 a_{7} \beta^{3} \\
& \left.-3 \beta^{3} b_{6}+4 \beta^{2} b_{1}\right)+\sin ^{4} \theta \cos ^{4} \theta\left(20 \alpha^{6} a_{8}-15 \alpha^{6} b_{9}-5 \alpha^{5} a_{5}\right. \\
& +17 \alpha^{4} a_{8} \beta+15 \alpha^{4} \beta b_{9}-22 \alpha^{3} a_{5} \beta-6 \alpha^{2} a_{8} \beta^{2}+27 \alpha^{2} \beta^{2} b_{9}-9 \alpha a_{5} \beta^{2} \\
& \left.-3 a_{8} \beta^{3}-3 \beta^{3} b_{9}\right) \text {, } \\
& A_{8}=\sqrt{\alpha^{2}-\beta R^{2} \cos ^{2} \theta+\beta}\left(\alpha a_{9}\left(15 \alpha^{4}-10 \alpha^{2} \beta-9 \beta^{2}\right) \sin ^{5} \theta \cos ^{4} \theta\right. \\
& +\sin ^{2} \theta \cos ^{7} \theta\left(15 \alpha^{5} a_{6}+9 \alpha^{5} a_{8}-6 \alpha^{5} b_{7}-9 \alpha^{4} a_{3}-4 \alpha^{4} a_{5}+5 \alpha^{4} b_{4}\right. \\
& +4 \alpha^{3} a_{1}-10 \alpha^{3} a_{6} \beta+10 \alpha^{3} a_{8} \beta+20 \alpha^{3} \beta b_{7}-4 \alpha^{3} b_{2}-6 \alpha^{2} a_{3} \beta \\
& -16 \alpha^{2} a_{5} \beta-10 \alpha^{2} \beta b_{4}+8 \alpha a_{1} \beta-9 \alpha a_{6} \beta^{2}-15 \alpha a_{8} \beta^{2}-6 \alpha \beta^{2} b_{7} \\
& \left.+4 \alpha \beta b_{2}+3 a_{3} \beta^{2}+4 a_{5} \beta^{2}+\beta^{2} b_{4}\right)+\cos ^{9} \theta\left(9 \alpha^{5} a_{6}-4 \alpha^{4} a_{3}\right. \\
& \left.+10 \alpha^{3} a_{6} \beta-16 \alpha^{2} a_{3} \beta+12 \alpha a_{1} \beta-15 \alpha a_{6} \beta^{2}+4 a_{3} \beta^{2}\right) \\
& +\sin ^{3} \theta \cos ^{6} \theta\left(15 \alpha^{5} a_{7}+9 \alpha^{5} a_{9}-6 \alpha^{5} b_{8}-9 \alpha^{4} a_{4}+5 \alpha^{4} b_{5}+4 \alpha^{3} a_{2}\right. \\
& -10 \alpha^{3} a_{7} \beta+10 \alpha^{3} a_{9} \beta+20 \alpha^{3} \beta b_{8}-6 \alpha^{2} a_{4} \beta-10 \alpha^{2} \beta b_{5}+8 \alpha a_{2} \beta \\
& \left.-9 \alpha a_{7} \beta^{2}-15 \alpha a_{9} \beta^{2}-6 \alpha \beta^{2} b_{8}+3 a_{4} \beta^{2}+\beta^{2} b_{5}\right) \\
& +\sin \theta \cos ^{8} \theta\left(9 \alpha^{5} a_{7}-6 \alpha^{5} b_{6}-4 \alpha^{4} a_{4}+5 \alpha^{4} b_{3}+10 \alpha^{3} a_{7} \beta\right. \\
& +20 \alpha^{3} \beta b_{6}-4 \alpha^{3} b_{1}-16 \alpha^{2} a_{4} \beta-10 \alpha^{2} \beta b_{3}+12 \alpha a_{2} \beta-15 \alpha a_{7} \beta^{2} \\
& \left.-6 \alpha \beta^{2} b_{6}+4 \alpha \beta b_{1}+4 a_{4} \beta^{2}+\beta^{2} b_{3}\right)+\sin ^{4} \theta \cos ^{5} \theta\left(15 \alpha^{5} a_{8}\right. \\
& -6 \alpha^{5} b_{9}-9 \alpha^{4} a_{5}-10 \alpha^{3} a_{8} \beta+20 \alpha^{3} \beta b_{9}-6 \alpha^{2} a_{5} \beta-9 \alpha a_{8} \beta^{2} \\
& \left.\left.-6 \alpha \beta^{2} b_{9}+3 a_{5} \beta^{2}\right)\right) \text {, } \\
& A_{9}=a_{9}\left(6 \alpha^{6}-25 \alpha^{4} \beta-12 \alpha^{2} \beta^{2}+3 \beta^{3}\right) \sin ^{5} \theta \cos ^{5} \theta+\sin ^{2} \theta \cos ^{8} \theta\left(6 \alpha^{6} a_{6}\right. \\
& +5 \alpha^{6} a_{8}-\alpha^{6} b_{7}-5 \alpha^{5} a_{3}-4 \alpha^{5} a_{5}+\alpha^{5} b_{4}+4 \alpha^{4} a_{1}-25 \alpha^{4} a_{6} \beta \\
& -10 \alpha^{4} a_{8} \beta+15 \alpha^{4} \beta b_{7}-\alpha^{4} b_{2}+6 \alpha^{3} a_{3} \beta-4 \alpha^{3} a_{5} \beta-10 \alpha^{3} \beta b_{4} \\
& +4 \alpha^{2} a_{1} \beta-12 \alpha^{2} a_{6} \beta^{2}-27 \alpha^{2} a_{8} \beta^{2}-15 \alpha^{2} \beta^{2} b_{7}+6 \alpha^{2} \beta b_{2}+11 \alpha a_{3} \beta^{2} \\
& \left.+16 \alpha a_{5} \beta^{2}+5 \alpha \beta^{2} b_{4}-4 a_{1} \beta^{2}+3 a_{6} \beta^{3}+4 a_{8} \beta^{3}+\beta^{3} b_{7}-\beta^{2} b_{2}\right) \\
& +\cos ^{10} \theta\left(5 \alpha^{6} a_{6}-4 \alpha^{5} a_{3}+3 \alpha^{4} a_{1}-10 \alpha^{4} a_{6} \beta-4 \alpha^{3} a_{3} \beta+10 \alpha^{2} a_{1} \beta\right. \\
& \left.-27 \alpha^{2} a_{6} \beta^{2}+16 \alpha a_{3} \beta^{2}-5 a_{1} \beta^{2}+4 a_{6} \beta^{3}\right)+\sin ^{3} \theta \cos ^{7} \theta\left(6 \alpha^{6} a_{7}\right. \\
& +5 \alpha^{6} a_{9}-\alpha^{6} b_{8}-5 \alpha^{5} a_{4}+\alpha^{5} b_{5}+4 \alpha^{4} a_{2}-25 \alpha^{4} a_{7} \beta-10 \alpha^{4} a_{9} \beta \\
& +15 \alpha^{4} \beta b_{8}+6 \alpha^{3} a_{4} \beta-10 \alpha^{3} \beta b_{5}+4 \alpha^{2} a_{2} \beta-12 \alpha^{2} a_{7} \beta^{2}-27 \alpha^{2} a_{9} \beta^{2} \\
& \left.-15 \alpha^{2} \beta^{2} b_{8}+11 \alpha a_{4} \beta^{2}+5 \alpha \beta^{2} b_{5}-4 a_{2} \beta^{2}+3 a_{7} \beta^{3}+4 a_{9} \beta^{3}+\beta^{3} b_{8}\right) \\
& +\sin \theta \cos ^{9} \theta\left(5 \alpha^{6} a_{7}-\alpha^{6} b_{6}-4 \alpha^{5} a_{4}+\alpha^{5} b_{3}+3 \alpha^{4} a_{2}-10 \alpha^{4} a_{7} \beta\right. \\
& +15 \alpha^{4} \beta b_{6}-\alpha^{4} b_{1}-4 \alpha^{3} a_{4} \beta-10 \alpha^{3} \beta b_{3}+10 \alpha^{2} a_{2} \beta-27 \alpha^{2} a_{7} \beta^{2} \\
& -15 \alpha^{2} \beta^{2} b_{6}+6 \alpha^{2} \beta b_{1}+16 \alpha a_{4} \beta^{2}+5 \alpha \beta^{2} b_{3}-5 a_{2} \beta^{2}+4 a_{7} \beta^{3}+\beta^{3} b_{6} \\
& \left.-\beta^{2} b_{1}\right)+\sin ^{4} \theta \cos ^{6} \theta\left(6 \alpha^{6} a_{8}-\alpha^{6} b_{9}-5 \alpha^{5} a_{5}-25 \alpha^{4} a_{8} \beta+15 \alpha^{4} \beta b_{9}\right. \\
& \left.+6 \alpha^{3} a_{5} \beta-12 \alpha^{2} a_{8} \beta^{2}-15 \alpha^{2} \beta^{2} b_{9}+11 \alpha a_{5} \beta^{2}+3 a_{8} \beta^{3}+\beta^{3} b_{9}\right), \\
& A_{10}=\sqrt{\alpha^{2}-\beta R^{2} \cos ^{2} \theta+\beta}\left(\alpha a_{9}\left(\alpha^{4}-10 \alpha^{2} \beta+5 \beta^{2}\right) \sin ^{5} \theta \cos ^{6} \theta\right.
\end{aligned}
$$




$$
\begin{aligned}
+ & \sin ^{2} \theta \cos ^{9} \theta\left(\alpha^{5} a_{6}+\alpha^{5} a_{8}-\alpha^{4} a_{3}-\alpha^{4} a_{5}+\alpha^{3} a_{1}-10 \alpha^{3} a_{6} \beta\right. \\
- & 10 \alpha^{3} a_{8} \beta+6 \alpha^{2} a_{3} \beta+6 \alpha^{2} a_{5} \beta-3 \alpha a_{1} \beta+5 \alpha a_{6} \beta^{2}+5 \alpha a_{8} \beta^{2}-a_{3} \beta^{2} \\
- & \left.a_{5} \beta^{2}\right)+\cos ^{11} \theta\left(\alpha^{5} a_{6}-\alpha^{4} a_{3}+\alpha^{3} a_{1}-10 \alpha^{3} a_{6} \beta+6 \alpha^{2} a_{3} \beta\right. \\
- & \left.3 \alpha a_{1} \beta+5 \alpha a_{6} \beta^{2}-a_{3} \beta^{2}\right)+\sin ^{3} \theta \cos ^{8} \theta\left(\alpha^{5} a_{7}+\alpha^{5} a_{9}-\alpha^{4} a_{4}\right. \\
+ & \alpha^{3} a_{2}-10 \alpha^{3} a_{7} \beta-10 \alpha^{3} a_{9} \beta+6 \alpha^{2} a_{4} \beta-3 \alpha a_{2} \beta+5 \alpha a_{7} \beta^{2}+5 \alpha a_{9} \beta^{2} \\
- & \left.a_{4} \beta^{2}\right)+\sin \theta \cos ^{10} \theta\left(\alpha^{5} a_{7}-\alpha^{4} a_{4}+\alpha^{3} a_{2}-10 \alpha^{3} a_{7} \beta+6 \alpha^{2} a_{4} \beta\right. \\
- & \left.3 \alpha a_{2} \beta+5 \alpha a_{7} \beta^{2}-a_{4} \beta^{2}\right)+\sin ^{4} \theta \cos ^{7} \theta\left(\alpha^{5} a_{8}-\alpha^{4} a_{5}-10 \alpha^{3} a_{8} \beta\right. \\
& \left.\left.+6 \alpha^{2} a_{5} \beta+5 \alpha a_{8} \beta^{2}-a_{5} \beta^{2}\right)\right) \\
A_{11} & -a_{9} \beta\left(5 \alpha^{4}-10 \alpha^{2} \beta+\beta^{2}\right) \sin ^{5} \theta \cos ^{7} \theta-\beta \sin ^{2} \theta \cos ^{10} \theta\left(5 \alpha^{4} a_{6}\right. \\
& +5 \alpha^{4} a_{8}-4 \alpha^{3} a_{3}-4 \alpha^{3} a_{5}+3 \alpha^{2} a_{1}-10 \alpha^{2} a_{6} \beta-10 \alpha^{2} a_{8} \beta+4 \alpha a_{3} \beta \\
& \left.+4 \alpha a_{5} \beta-a_{1} \beta+a_{6} \beta^{2}+a_{8} \beta^{2}\right)-\beta \cos ^{12} \theta\left(5 \alpha^{4} a_{6}-4 \alpha^{3} a_{3}+3 \alpha^{2} a_{1}\right. \\
& \left.-10 \alpha^{2} a_{6} \beta+4 \alpha a_{3} \beta-a_{1} \beta+a_{6} \beta^{2}\right)-\beta \sin ^{3} \theta \cos ^{9} \theta\left(5 \alpha^{4} a_{7}+5 \alpha^{4} a_{9}\right. \\
& -4 \alpha^{3} a_{4}+3 \alpha^{2} a_{2}-10 \alpha^{2} a_{7} \beta-10 \alpha^{2} a_{9} \beta+4 \alpha a_{4} \beta-a_{2} \beta+a_{7} \beta^{2} \\
& \left.+a_{9} \beta^{2}\right)-\beta \sin \theta \cos ^{11} \theta\left(5 \alpha^{4} a_{7}-4 \alpha^{3} a_{4}+3 \alpha^{2} a_{2}-10 \alpha^{2} a_{7} \beta+4 \alpha a_{4} \beta\right. \\
& \left.-a_{2} \beta+a_{7} \beta^{2}\right)-\beta \sin ^{4} \theta \cos ^{8} \theta\left(5 \alpha^{4} a_{8}-4 \alpha^{3} a_{5}-10 \alpha^{2} a_{8} \beta+4 \alpha a_{5} \beta\right. \\
& \left.+a_{8} \beta^{2}\right) \\
Q_{1}= & 2(R \cos \theta-1)(R \cos \theta+1)\left(\alpha R \cos \theta \sqrt{\alpha^{2}-\beta R^{2} \cos ^{2} \theta+\beta}+\alpha^{2}\right. \\
& \left.-\beta R^{2} \cos ^{2} \theta+\beta\right)\left(2 \alpha R \cos ^{2} \theta \sqrt{\alpha^{2}-\beta R^{2} \cos ^{2} \theta+\beta}+R^{2}\left(\alpha^{2}\right.\right. \\
& \left.-\beta) \cos ^{2} \theta+\alpha^{2}+\beta\right)^{2} .
\end{aligned}
$$

Integrating the right part of differential equation (13) we get the averaging function $f:(0,1) \rightarrow \mathbb{R}$ given by

$$
\begin{aligned}
f(R)= & f_{0}\left[\frac{\left(\alpha^{2} a_{6}-3 \alpha^{2} a_{8}-\alpha^{2} b_{7}+3 \alpha^{2} b_{9}+a_{1}+a_{6} \beta-3 a_{8} \beta\right.}{4\left(\alpha^{2}+\beta\right)}\right. \\
& \left.+\frac{\left.-\beta b_{7}+3 \beta b_{9}+b_{2}\right)}{4\left(\alpha^{2}+\beta\right)}\right]+f_{1}\left[\frac{\left(\alpha^{4} a_{6}+\alpha^{4} a_{8}-\alpha^{3} a_{3}-\alpha^{3} a_{5}+\alpha^{2} a_{1}\right.}{4\left(\alpha^{2}+\beta\right)^{2}}\right. \\
& \left.+\frac{\left.2 \alpha^{2} a_{6} \beta+2 \alpha^{2} a_{8} \beta-\alpha a_{3} \beta-\alpha a_{5} \beta-a_{1} \beta+a_{6} \beta^{2}+a_{8} \beta^{2}\right)}{4\left(\alpha^{2}+\beta\right)^{2}}\right] \\
& +\frac{1}{2} f_{2}\left(a_{8}-b_{9}\right)+f_{3}\left(a_{6}-a_{8}-b_{7}+b_{9}\right),
\end{aligned}
$$

where

$$
f_{0}=R, \quad f_{1}=R^{3}, \quad f_{2}=R \sqrt{1-R^{2}} \quad \text { and } \quad f_{3}=\sqrt{1-R^{2}} / R .
$$

We have to study the number of simple zeros of averaging function $f$. For this we shall calculate the Wronskians $W\left(f_{0}, \ldots, f_{k}\right)$, for $k=0, \ldots, 3$ and 
prove that they do not vanish for $R \in(0,1)$. In fact,

$$
\begin{gathered}
W\left(f_{0}\right)=R, \quad W\left(f_{0}, f_{1}\right)=2 R^{3}, \quad W\left(f_{0}, f_{1}, f_{2}\right)=-\frac{2 R^{6}}{\left(1-R^{2}\right)^{3 / 2}}, \\
W\left(f_{0}, \ldots, f_{3}\right)=-\frac{12 R^{2}\left(R^{4}+4\left(\sqrt{1-R^{2}}-2\right) R^{2}-8 \sqrt{1-R^{2}}+8\right)}{\left(R^{2}-1\right)^{3}} .
\end{gathered}
$$

Obviously the first three Wronskians do not vanish. The $W\left(f_{0}, \ldots, f_{3}\right)$ is equal to zero if and only if

$$
R^{4}-8 R^{2}+4 \sqrt{1-R^{2}}\left(R^{2}-2\right)+8=0
$$

Passing the term $4 \sqrt{1-R^{2}}\left(R^{2}-2\right)$ to the right hand side of the previous equality and taking the square in both sides we obtain $R^{8}=0$ which is it impossible because $R \in(0,1)$. Thus the Wronskians $W\left(f_{0}, . ., f_{k}\right) \neq 0$ for $k=0, . ., 3$. Hence, since $\left(f_{0}, \ldots, f_{3}\right)$ is an ECT-system the averaging function $f$ has at most 3 simple zeros and they are reached. By Theorem 4, these zeros provide 3 limit cycles for system (1).

3.2. Proof for the class $P_{4}$. Suppose that $\alpha<0$ and $\beta>0$. The positive function $\rho$ that satisfies $H(\rho \cos \theta, \rho \sin \theta)=R^{2}$ is $\rho:(0, \sqrt{-\alpha / \beta}) \rightarrow \mathbb{R}$ given by

$$
\rho=\frac{\beta R}{\sqrt{\beta \cos ^{2} \theta-\sin ^{2} \theta\left(\alpha+\beta R^{2}\right)}} .
$$

The integrating factor

$$
\mu(x, y)=-\frac{2}{\left(\beta+y^{2}\right)^{2}}
$$

corresponds to the first integral

$$
H(x, y)=-\frac{\alpha y^{2}-\beta x^{2}}{\beta\left(\beta+y^{2}\right)}
$$

because they satisfy $\mu P=H_{y}$ and $\mu Q=-H_{x}$. We transform system (4) using Theorem 5 in the following averaging standard form

$$
\frac{d R}{d \varphi}=\varepsilon \sum_{i=1}^{7} \frac{B_{i}(\varphi, \alpha, \beta, a, b)}{Q_{2}(R, \varphi, \alpha, \beta)} R^{i}+\mathcal{O}\left(\varepsilon^{2}\right)
$$


where $a=\left(a_{1}, \ldots, a_{9}\right), b=\left(b_{1}, \ldots, b_{9}\right)$ and

$$
\begin{aligned}
& B_{1}=\alpha^{3} b_{2} \sin ^{6} \theta-\alpha^{2} \beta\left(a_{1}+2 b_{2}\right) \sin ^{4} \theta \cos ^{2} \theta+\alpha^{2} \sin ^{5} \theta \cos \theta\left(\alpha b_{1}-a_{2} \beta\right) \\
& +\alpha \beta^{2}\left(2 a_{1}+b_{2}\right) \sin ^{2} \theta \cos ^{4} \theta-\beta^{2} \sin \theta \cos ^{5} \theta\left(a_{2} \beta-\alpha b_{1}\right), \\
& -2 \alpha \beta \sin ^{3} \theta \cos ^{3} \theta\left(\alpha b_{1}-a_{2} \beta\right)-a_{1} \beta^{3} \cos ^{6} \theta \\
& B_{2}=\sqrt{-\alpha \sin ^{2} \theta+\beta \cos ^{2} \theta-\beta R^{2} \sin ^{2} \theta}\left(-\alpha^{2} \beta b_{5} \sin ^{5} \theta\right. \\
& -\beta^{2} \sin ^{2} \theta \cos ^{3} \theta\left(-\alpha a_{3}-\alpha b_{4}+a_{5} \beta\right)-\beta^{2} \sin \theta \cos ^{4} \theta\left(a_{4} \beta-\alpha b_{3}\right) \\
& +\alpha \beta \sin ^{3} \theta \cos ^{2} \theta\left(-\alpha b_{3}+a_{4} \beta+\beta b_{5}\right)+\alpha \beta \sin ^{4} \theta \cos \theta\left(a_{5} \beta-\alpha b_{4}\right) \\
& \left.-a_{3} \beta^{3} \cos ^{5} \theta\right) \text {, } \\
& B_{3}=\beta^{2} \sin ^{3} \theta \cos ^{3} \theta\left(-\alpha^{2} b_{6}+\alpha a_{7} \beta+\alpha \beta b_{8}-4 \alpha b_{1}+2 a_{2} \beta-a_{9} \beta^{2}\right) \\
& -\alpha^{2} \beta \sin ^{6} \theta\left(\beta b_{9}-3 b_{2}\right)+\beta^{3} \sin ^{2} \theta \cos ^{4} \theta\left(\alpha a_{6}+\alpha b_{7}+2 a_{1}-a_{8} \beta\right. \\
& \left.+b_{2}\right)-\alpha \beta^{2} \sin ^{4} \theta \cos ^{2} \theta\left(\alpha b_{7}+2 a_{1}-a_{8} \beta-\beta b_{9}+4 b_{2}\right) \\
& -\alpha \beta \sin ^{5} \theta \cos \theta\left(\alpha \beta b_{8}-3 \alpha b_{1}+2 a_{2} \beta-a_{9} \beta^{2}\right)-\beta^{3} \sin \theta \cos ^{5} \theta\left(-\alpha b_{6}\right. \\
& \left.+a_{7} \beta-b_{1}\right)-a_{6} \beta^{4} \cos ^{6} \theta, \\
& B_{4}=\sqrt{-\alpha \sin ^{2} \theta+\beta \cos ^{2} \theta-\beta R^{2} \sin ^{2} \theta}\left(\beta ^ { 2 } \operatorname { s i n } ^ { 3 } \theta \operatorname { c o s } ^ { 2 } \theta \left(-2 \alpha b_{3}+a_{4} \beta\right.\right. \\
& \left.+\beta b_{5}\right)+\beta^{2} \sin ^{4} \theta \cos \theta\left(a_{5} \beta-2 \alpha b_{4}\right)-2 \alpha \beta^{2} b_{5} \sin ^{5} \theta+\beta^{3}\left(a_{3}\right. \\
& \left.\left.+b_{4}\right) \sin ^{2} \theta \cos ^{3} \theta+\beta^{3} b_{3} \sin \theta \cos ^{4} \theta\right) \text {, } \\
& B_{5}=-\beta^{3} \sin ^{4} \theta \cos ^{2} \theta\left(2 \alpha b_{7}+a_{1}-a_{8} \beta-\beta b_{9}+2 b_{2}\right) \\
& -\beta^{2} \sin ^{5} \theta \cos \theta\left(2 \alpha \beta b_{8}-3 \alpha b_{1}+a_{2} \beta-a_{9} \beta^{2}\right) \\
& +\beta^{3} \sin ^{3} \theta \cos ^{3} \theta\left(-2 \alpha b_{6}+a_{7} \beta+\beta b_{8}-2 b_{1}\right)-\alpha \beta^{2} \sin ^{6} \theta\left(2 \beta b_{9}-3 b_{2}\right) \\
& +\beta^{4}\left(a_{6}+b_{7}\right) \sin ^{2} \theta \cos ^{4} \theta+\beta^{4} b_{6} \sin \theta \cos ^{5} \theta \text {, } \\
& B_{6}=\sqrt{-\alpha \sin ^{2} \theta+\beta \cos ^{2} \theta-\beta R^{2} \sin ^{2} \theta}\left(-\beta^{3} b_{3} \sin ^{3} \theta \cos ^{2} \theta\right. \\
& \left.-\beta^{3} b_{4} \sin ^{4} \theta \cos \theta-\beta^{3} b_{5} \sin ^{5} \theta\right) \text {, } \\
& B_{7}=-\beta^{4} b_{6} \sin ^{3} \theta \cos ^{3} \theta-\beta^{4} b_{7} \sin ^{4} \theta \cos ^{2} \theta-\beta^{3} \sin ^{5} \theta \cos \theta\left(\beta b_{8}-b_{1}\right) \\
& -\beta^{3} \sin ^{6} \theta\left(\beta b_{9}-b_{2}\right), \\
& Q_{2}=\left(\beta \cos ^{2} \theta-\alpha \sin ^{2} \theta\right)^{2}\left(\beta \cos ^{2} \theta-\sin ^{2} \theta\left(\alpha+\beta R^{2}\right)\right)^{2} \text {. }
\end{aligned}
$$

Integrating the right part of differential equation (14) we get the function $f:(0, \sqrt{-\alpha / \beta})$ given by

$$
\begin{aligned}
f= & \alpha f_{0} \frac{\left(3 \alpha a_{6}-3 \alpha b_{7}-a_{1}+a_{8} \beta-\beta b_{9}-b_{2}\right)}{2(-\alpha)^{3 / 2} \sqrt{\beta}}+f_{1} \frac{\sqrt{\beta}\left(-\alpha b_{7}+\beta b_{9}-b_{2}\right)}{2(-\alpha)^{3 / 2}} \\
& -f_{2} \frac{\left(a_{6}-b_{7}\right)}{\sqrt{\beta}}+f_{3} \frac{\left(\alpha a_{6}-\alpha b_{7}+a_{8} \beta-\beta b_{9}\right)}{\beta^{3 / 2}}
\end{aligned}
$$

where

$f_{0}=R, \quad f_{1}=R^{3}, \quad f_{2}=-R \sqrt{-\alpha-\beta R^{2}} \quad$ and $\quad f_{3}=\frac{\sqrt{-\alpha}-\sqrt{-\alpha-\beta R^{2}}}{R}$. 
We have

$$
\begin{array}{r}
W\left(f_{0}\right)=R, \quad W\left(f_{0}, f_{1}\right)=2 R^{3}, \quad W\left(f_{0}, f_{1}, f_{2}\right)=\frac{2 \beta^{2} R^{6}}{\left(-\alpha-\beta R^{2}\right)^{3 / 2}}, \\
W\left(f_{0}, \ldots, f_{3}\right)=-\frac{12 \beta^{2} R^{2}\left(8 \alpha^{2}+8 \alpha \beta R^{2}+4 \sqrt{-\alpha} \sqrt{-\alpha-\beta R^{2}}\left(2 \alpha+\beta R^{2}\right)+\beta^{2} R^{4}\right)}{\left(\alpha+\beta R^{2}\right)^{3}}
\end{array}
$$

Clearly the first three Wronskians do not vanish. The last Wronskian is equal to zero if and only if

$$
8 \alpha^{2}+8 \alpha \beta R^{2}+b^{2} R^{4}+4 \sqrt{-\alpha} \sqrt{-\alpha-\beta R^{2}}\left(2 \alpha+\beta R^{2}\right)=0 .
$$

Passing the term $4 \sqrt{-\alpha} \sqrt{-\alpha-\beta R^{2}}\left(2 \alpha+\beta R^{2}\right)$ to the right hand side of the previous equality and taking the square in both sides we get $\beta^{4} R^{8}=$ 0 . This is impossible because $R \in(0, \sqrt{-\alpha / \beta})$. Thus the Wronskians $W\left(f_{0}, . ., f_{k}\right), k=0, \ldots, 3$ do not vanish for $R \in(0, \sqrt{-\alpha / \beta})$. Therefore, since that $\left(f_{0}, \ldots, f_{3}\right)$ is an ECT-system the averaging function $f$ has at most 3 simple zeros and they are reached. By Theorem 4 , these zeros provide 3 limit cycles for system (4). If $\alpha>0$ and $\beta<0$ then we take $\bar{\rho}=-\rho$ and the result obtained is analogous.

3.3. Proof for the class $P_{6}$. Again the integrating factor

$$
\mu(x, y)=\frac{1}{(\alpha+x)^{4}}
$$

corresponds to the first integral

$$
H(x, y)=\frac{x^{2}+y^{2}}{(\alpha+x)^{2}}
$$

because $\mu P=H_{y}$ and $\mu Q=-H_{x}$. Again by solving implicitly the equation $H(\rho \cos \theta, \rho \cos \theta)=R^{2}$ we get the positive function $\rho:(0,1) \rightarrow \mathbb{R}$ given by

$$
\rho=-\frac{2 R\left(\sqrt{\alpha^{2}}+\alpha R \cos \theta\right)}{R^{2}\left(2 \cos ^{2} \theta-1\right)+R^{2}-2} .
$$

We transform system (6) using Theorem 5 in the following averaging standard form

$$
\frac{d R}{d \varphi}=\varepsilon \frac{N(\varphi, \alpha, \beta, a, b)}{Q_{3}(R, \varphi, \alpha, \beta)}+\mathcal{O}\left(\varepsilon^{2}\right)
$$


where $a=\left(a_{1}, \ldots, a_{9}\right), b=\left(b_{1}, \ldots, b_{9}\right)$ and

$$
\begin{aligned}
& N=R\left(\alpha R \cos \theta+\sqrt{\alpha^{2}}\right)^{2}\left(\alpha a_{1} R^{6} \cos ^{8} \theta-\alpha^{2} a_{3} R^{6} \cos ^{8} \theta+\alpha^{3} a_{6} R^{6} \cos ^{8} \theta\right. \\
& -\alpha \sqrt{\alpha^{2}} a_{3} R^{5} \cos ^{7} \theta+2\left(\alpha^{2}\right)^{3 / 2} a_{6} R^{5} \cos ^{7} \theta+\alpha a_{2} R^{6} \sin \theta \cos ^{7} \theta \\
& -\alpha^{2} a_{4} R^{6} \sin \theta \cos ^{7} \theta+\alpha^{3} a_{7} R^{6} \sin \theta \cos ^{7} \theta-3 \alpha a_{1} R^{4} \cos ^{6} \theta \\
& +2 \alpha^{2} a_{3} R^{4} \cos ^{6} \theta+\alpha a_{1} R^{6} \sin ^{2} \theta \cos ^{6} \theta-\alpha^{2} a_{3} R^{6} \sin ^{2} \theta \cos ^{6} \theta \\
& -\alpha^{2} a_{5} R^{6} \sin ^{2} \theta \cos ^{6} \theta+\alpha^{3} a_{6} R^{6} \sin ^{2} \theta \cos ^{6} \theta+\alpha^{3} a_{8} R^{6} \sin ^{2} \theta \cos ^{6} \theta \\
& -\alpha \sqrt{\alpha^{2}} a_{4} R^{5} \sin \theta \cos ^{6} \theta+2\left(\alpha^{2}\right)^{3 / 2} a_{7} R^{5} \sin \theta \cos ^{6} \theta \\
& -\sqrt{\alpha^{2}} b_{1} R^{5} \sin \theta \cos ^{6} \theta+\alpha \sqrt{\alpha^{2}} b_{3} R^{5} \sin \theta \cos ^{6} \theta \\
& -\left(\alpha^{2}\right)^{3 / 2} b_{6} R^{5} \sin \theta \cos ^{6} \theta+2 \alpha \sqrt{\alpha^{2}} a_{3} R^{3} \cos ^{5} \theta-2\left(\alpha^{2}\right)^{3 / 2} a_{6} R^{3} \cos ^{5} \theta \\
& +\alpha a_{2} R^{6} \sin ^{3} \theta \cos ^{5} \theta-\alpha^{2} a_{4} R^{6} \sin ^{3} \theta \cos ^{5} \theta+\alpha^{3} a_{7} R^{6} \sin ^{3} \theta \cos ^{5} \theta \\
& +\alpha^{3} a_{9} R^{6} \sin ^{3} \theta \cos ^{5} \theta+\sqrt{\alpha^{2}} a_{1} R^{5} \sin ^{2} \theta \cos ^{5} \theta-2 \alpha \sqrt{\alpha^{2}} a_{3} R^{5} \sin ^{2} \theta \cos ^{5} \theta \\
& -\alpha \sqrt{\alpha^{2}} a_{5} R^{5} \sin ^{2} \theta \cos ^{5} \theta+3\left(\alpha^{2}\right)^{3 / 2} a_{6} R^{5} \sin ^{2} \theta \cos ^{5} \theta \\
& +2\left(\alpha^{2}\right)^{3 / 2} a_{8} R^{5} \sin ^{2} \theta \cos ^{5} \theta-\sqrt{\alpha^{2}} b_{2} R^{5} \sin ^{2} \theta \cos ^{5} \theta \\
& +\alpha \sqrt{\alpha^{2}} b_{4} R^{5} \sin ^{2} \theta \cos ^{5} \theta-\left(\alpha^{2}\right)^{3 / 2} b_{7} R^{5} \sin ^{2} \theta \cos ^{5} \theta \\
& -3 \alpha a_{2} R^{4} \sin \theta \cos ^{5} \theta+2 \alpha^{2} a_{4} R^{4} \sin \theta \cos ^{5} \theta-\alpha b_{1} R^{4} \sin \theta \cos ^{5} \theta \\
& +2 \alpha^{2} b_{3} R^{4} \sin \theta \cos ^{5} \theta-3 \alpha^{3} b_{6} R^{4} \sin \theta \cos ^{5} \theta-\alpha^{2} a_{5} R^{6} \sin ^{4} \theta \cos ^{4} \theta \\
& +\alpha^{3} a_{8} R^{6} \sin ^{4} \theta \cos ^{4} \theta+\sqrt{\alpha^{2}} a_{2} R^{5} \sin ^{3} \theta \cos ^{4} \theta-2 \alpha \sqrt{\alpha^{2}} a_{4} R^{5} \sin ^{3} \theta \cos ^{4} \theta \\
& +3\left(\alpha^{2}\right)^{3 / 2} a_{7} R^{5} \sin ^{3} \theta \cos ^{4} \theta+2\left(\alpha^{2}\right)^{3 / 2} a_{9} R^{5} \sin ^{3} \theta \cos ^{4} \theta \\
& +\alpha \sqrt{\alpha^{2}} b_{5} R^{5} \sin ^{3} \theta \cos ^{4} \theta-\left(\alpha^{2}\right)^{3 / 2} b_{8} R^{5} \sin ^{3} \theta \cos ^{4} \theta+3 \alpha a_{1} R^{2} \cos ^{4} \theta \\
& -\alpha^{2} a_{3} R^{2} \cos ^{4} \theta-\alpha^{3} a_{6} R^{2} \cos ^{4} \theta-2 \alpha a_{1} R^{4} \sin ^{2} \theta \cos ^{4} \theta \\
& +2 \alpha^{2} a_{5} R^{4} \sin ^{2} \theta \cos ^{4} \theta+3 \alpha^{3} a_{6} R^{4} \sin ^{2} \theta \cos ^{4} \theta-\alpha b_{2} R^{4} \sin ^{2} \theta \cos ^{4} \theta \\
& +2 \alpha^{2} b_{4} R^{4} \sin ^{2} \theta \cos ^{4} \theta-3 \alpha^{3} b_{7} R^{4} \sin ^{2} \theta \cos ^{4} \theta+2 \alpha \sqrt{\alpha^{2}} a_{4} R^{3} \sin \theta \cos ^{4} \theta \\
& -2\left(\alpha^{2}\right)^{3 / 2} a_{7} R^{3} \sin \theta \cos ^{4} \theta+2 \sqrt{\alpha^{2}} b_{1} R^{3} \sin \theta \cos ^{4} \theta \\
& -3\left(\alpha^{2}\right)^{3 / 2} b_{6} R^{3} \sin \theta \cos ^{4} \theta+\alpha^{3} a_{9} R^{6} \sin ^{5} \theta \cos ^{3} \theta \\
& -2 \alpha \sqrt{\alpha^{2}} a_{5} R^{5} \sin ^{4} \theta \cos ^{3} \theta+3\left(\alpha^{2}\right)^{3 / 2} a_{8} R^{5} \sin ^{4} \theta \cos ^{3} \theta \\
& -\left(\alpha^{2}\right)^{3 / 2} b_{9} R^{5} \sin ^{4} \theta \cos ^{3} \theta-2 \alpha a_{2} R^{4} \sin ^{3} \theta \cos ^{3} \theta \\
& +3 \alpha^{3} a_{7} R^{4} \sin ^{3} \theta \cos ^{3} \theta+2 \alpha^{2} b_{5} R^{4} \sin ^{3} \theta \cos ^{3} \theta-3 \alpha^{3} b_{8} R^{4} \sin ^{3} \theta \cos ^{3} \theta \\
& -2 \sqrt{\alpha^{2}} a_{1} R^{3} \sin ^{2} \theta \cos ^{3} \theta+2 \alpha \sqrt{\alpha^{2}} a_{3} R^{3} \sin ^{2} \theta \cos ^{3} \theta \\
& +2 \alpha \sqrt{\alpha^{2}} a_{5} R^{3} \sin ^{2} \theta \cos ^{3} \theta+\left(\alpha^{2}\right)^{3 / 2} a_{6} R^{3} \sin ^{2} \theta \cos ^{3} \theta \\
& -2\left(\alpha^{2}\right)^{3 / 2} a_{8} R^{3} \sin ^{2} \theta \cos ^{3} \theta+2 \sqrt{\alpha^{2}} b_{2} R^{3} \sin ^{2} \theta \cos ^{3} \theta \\
& -3\left(\alpha^{2}\right)^{3 / 2} b_{7} R^{3} \sin ^{2} \theta \cos ^{3} \theta-\alpha \sqrt{\alpha^{2}} a_{3} R \cos ^{3} \theta+3 \alpha a_{2} R^{2} \sin \theta \cos ^{3} \theta \\
& -\alpha^{2} a_{4} R^{2} \sin \theta \cos ^{3} \theta-\alpha^{3} a_{7} R^{2} \sin \theta \cos ^{3} \theta+2 \alpha b_{1} R^{2} \sin \theta \cos ^{3} \theta
\end{aligned}
$$




$$
\begin{aligned}
& -2 \alpha^{2} b_{3} R^{2} \sin \theta \cos ^{3} \theta-\alpha^{3} b_{6} R^{2} \sin \theta \cos ^{3} \theta+3\left(\alpha^{2}\right)^{3 / 2} a_{9} R^{5} \sin ^{5} \theta \cos ^{2} \theta \\
+ & 3 \alpha^{3} a_{8} R^{4} \sin ^{4} \theta \cos ^{2} \theta-3 \alpha^{3} b_{9} R^{4} \sin ^{4} \theta \cos ^{2} \theta-2 \sqrt{\alpha^{2}} a_{2} R^{3} \sin ^{3} \theta \cos ^{2} \theta \\
& +2 \alpha \sqrt{\alpha^{2}} a_{4} R^{3} \sin ^{3} \theta \cos ^{2} \theta+\left(\alpha^{2}\right)^{3 / 2} a_{7} R^{3} \sin ^{3} \theta \cos ^{2} \theta \\
& -2\left(\alpha^{2}\right)^{3 / 2} a_{9} R^{3} \sin ^{3} \theta \cos ^{2} \theta-3\left(\alpha^{2}\right)^{3 / 2} b_{8} R^{3} \sin ^{3} \theta \cos ^{2} \theta \\
& +\alpha a_{1} R^{2} \sin ^{2} \theta \cos ^{2} \theta+\alpha^{2} a_{3} R^{2} \sin ^{2} \theta \cos ^{2} \theta-\alpha^{2} a_{5} R^{2} \sin ^{2} \theta \cos ^{2} \theta \\
& -\alpha^{3} a_{8} R^{2} \sin ^{2} \theta \cos ^{2} \theta+2 \alpha b_{2} R^{2} \sin ^{2} \theta \cos ^{2} \theta-2 \alpha^{2} b_{4} R^{2} \sin ^{2} \theta \cos ^{2} \theta \\
& -\alpha^{3} b_{7} R^{2} \sin ^{2} \theta \cos ^{2} \theta-\alpha a_{1} \cos ^{2} \theta-\alpha \sqrt{\alpha^{2}} a_{4} R \sin \theta \cos ^{2} \theta \\
& -\sqrt{\alpha^{2}} b_{1} R \sin \theta \cos ^{2} \theta-\alpha \sqrt{\alpha^{2}} b_{3} R \sin \theta \cos ^{2} \theta+3 \alpha^{3} a_{9} R^{4} \sin ^{5} \theta \cos \theta \\
& +2 \alpha \sqrt{\alpha^{2}} a_{5} R^{3} \sin ^{4} \theta \cos \theta+\left(\alpha^{2}\right)^{3 / 2} a_{8} R^{3} \sin ^{4} \theta \cos \theta \\
& -3\left(\alpha^{2}\right)^{3 / 2} b_{9} R^{3} \sin ^{4} \theta \cos \theta+\alpha a_{2} R^{2} \sin ^{3} \theta \cos \theta+\alpha^{2} a_{4} R^{2} \sin ^{3} \theta \cos \theta \\
& -\alpha^{3} a_{9} R^{2} \sin ^{3} \theta \cos \theta-2 \alpha^{2} b_{5} R^{2} \sin ^{3} \theta \cos \theta-\alpha^{3} b_{8} R^{2} \sin ^{3} \theta \cos \theta \\
& +\sqrt{\alpha^{2}} a_{1} R \sin ^{2} \theta \cos \theta-\alpha \sqrt{\alpha^{2}} a_{5} R \sin \sin ^{2} \theta \cos \theta-\sqrt{\alpha^{2}} b_{2} R \sin ^{2} \theta \cos \theta \\
& -\alpha \sqrt{\alpha^{2}} b_{4} R \sin ^{2} \theta \cos \theta-\alpha a_{2} \sin \theta \cos \theta-\alpha b_{1} \sin \theta \cos \theta \\
& +\left(\alpha^{2}\right)^{3 / 2} a_{9} R^{3} \sin ^{5} \theta+\alpha^{2} a_{5} R^{2} \sin ^{4} \theta-\alpha^{3} b_{9} R^{2} \sin { }^{4} \theta+\sqrt{\alpha^{2}} a_{2} R \sin ^{3} \theta \\
& \left.-\alpha \sqrt{\alpha^{2}} b_{5} R \sin ^{3} \theta-\alpha b_{2} \sin ^{2} \theta\right) \\
& 2 \alpha(R \cos \theta-1)(R \cos \theta+1)\left(\sqrt{\alpha^{2}} R \cos \theta+\alpha\right)^{4}
\end{aligned}
$$

Integrating the right part of differential equation (15) we obtain the averaging function $f:(0,1) \rightarrow \mathbb{R}$ defined by

$$
\begin{aligned}
f(R)= & f_{0}\left[\frac{\left(\alpha^{2} a_{6}-3 \alpha^{2} a_{8}\right.}{4 \alpha^{2}}+\frac{\left.-\alpha^{2} b_{7} b_{9}+3 \alpha^{2}+a_{1}+b_{2}\right)}{4 \alpha^{2}}\right] \\
& +f_{1} \frac{\left(\alpha^{2} a_{6}+\alpha^{2} a_{8}-\alpha a_{3}-\alpha a_{5}+a_{1}\right)}{4 \alpha^{2}}+\frac{1}{2} f_{2}\left(a_{8}-b_{9}\right) \\
& +f_{3} \frac{\left(a_{6}-a_{8}-b_{7}+b_{9}\right)}{2},
\end{aligned}
$$

where

$$
f_{0}=R, \quad f_{1}=R^{3}, \quad f_{2}=R \sqrt{1-R^{2}} \text { and } \quad\left(\sqrt{1-R^{2}}-1\right) / R .
$$

We have that

$$
\begin{array}{r}
W\left(f_{0}\right)=R, \quad W\left(f_{0}, f_{1}\right)=2 R^{3}, \quad W\left(f_{0}, f_{1}, f_{3}\right)=-\frac{2 R^{6}}{\left(1-R^{2}\right)^{3 / 2}}, \\
W\left(f_{0}, . ., f_{3}\right)=-12 R^{2} \frac{\left(R^{4}-8 R^{2}+8+4 \sqrt{1-R^{2}}\left(R^{2}-2\right)\right)}{\left(R^{2}-1\right)^{3}} .
\end{array}
$$


The first three Wronskians do not vanish for $R \in(0,1)$. The last Wronskian is equal to zero if and only if

$$
R^{4}-8 R^{2}+8+4 \sqrt{1-R^{2}}\left(R^{2}-2\right)=0
$$

Passing the term $4 \sqrt{1-R^{2}}\left(R^{2}-2\right)$ to the right hand side and taking the square in both sides we get $R^{8}=0$. This is impossible because $R \in(0,1)$. Thus the Wronskians $W\left(f_{0}, . ., f_{k}\right), k=0, \ldots, 3$ do not vanish for $R \in(0,1)$. Therefore, since that $\left(f_{0}, \ldots, f_{3}\right)$ is an ECT-system the averaging function $f$ has at most 3 simple zeros and they are reached. By Theorem 4 , these zeros provide 3 limit cycles for system (6).

\section{Proof of Theorem 2}

At first we suppose that $\alpha^{2}+\beta<0$. As before we get the positive function $\rho:\left(0, \sqrt{-\alpha^{2} / \beta}\right) \rightarrow \mathbb{R}$ given by

$$
\rho=\frac{\beta^{2} R\left(\alpha^{2}+\beta\right)}{\alpha \beta^{2} R \cos \theta-\sqrt{\alpha^{2} \beta^{2}\left(\beta\left(\alpha^{2}+\beta\right) \sin ^{2} \theta-\alpha^{2} \cos ^{2} \theta\left(\alpha^{2}+\beta R^{2}+\beta\right)\right)}},
$$

by solving the implicit equation $H(\rho \cos \theta, \rho \sin \theta)=R^{2}$. The integrating factor

$$
\mu(x, y)=\frac{\alpha^{4}}{\left(\alpha^{2}\left(\beta+x^{2}\right)-2 \alpha \beta x+\beta^{2}\right)^{2}}
$$

corresponds to the first integral

$$
H(x, y)=\frac{\alpha^{2} \beta y^{2}-\alpha^{4} x^{2}}{\beta\left(\alpha^{2}\left(\beta+x^{2}\right)-2 \alpha \beta x+\beta^{2}\right)}
$$

because they satisfy $\mu P=H_{y}$ and $\mu Q=-H_{x}$. By using Theorem 5 , system 2 becomes

$$
\frac{d R}{d \varphi}=\varepsilon \sum_{i=1}^{5} \frac{C_{i}(\varphi, \alpha, \beta, a, b)}{Q_{4}(R, \varphi, \alpha, \beta)} R^{i}+\mathcal{O}\left(\varepsilon^{2}\right)
$$

where $a=\left(a_{1}, \ldots, a_{9}\right), b=\left(b_{1}, \ldots, b_{9}\right)$ and

$$
\begin{aligned}
C_{1}= & \left(\alpha^{2}+\beta\right)^{2} \sin ^{2} \theta \cos ^{6} \theta\left(\alpha^{2} a_{1}-2 a_{1} \beta-\beta b_{2}\right)+\alpha^{8} \beta^{2}\left(\alpha^{2}\right. \\
& +\beta)^{2} \sin \theta \cos ^{7} \theta\left(\alpha^{2} a_{2}-\beta b_{1}\right)-\alpha^{6} \beta^{3}\left(\alpha^{2}+\beta\right)^{2} \sin ^{4} \theta \cos ^{4} \theta\left(2 \alpha^{2} a_{1}\right. \\
& \left.+\alpha^{2} b_{2}-a_{1} \beta-2 \beta b_{2}\right)+\alpha^{6} \beta^{2}\left(\alpha^{2}-2 \beta\right)\left(\alpha^{2}+\beta\right)^{2} \sin ^{3} \theta \cos ^{5} \theta\left(\alpha^{2} a_{2}\right. \\
& \left.-\beta b_{1}\right)+\alpha^{4} \beta^{4}\left(\alpha^{2}+\beta\right)^{2} \sin ^{6} \theta \cos ^{2} \theta\left(\alpha^{2} a_{1}+2 \alpha^{2} b_{2}-\beta b_{2}\right) \\
& +\alpha^{4} \beta^{4}\left(\alpha^{2}+\beta\right)^{2} \sin ^{7} \theta \cos \theta\left(\alpha^{2} a_{2}-\beta b_{1}\right)-\alpha^{4} \beta^{3}\left(2 \alpha^{2}-\beta\right)\left(\alpha^{2}\right.
\end{aligned}
$$




$$
\begin{aligned}
& +\beta)^{2} \sin ^{5} \theta \cos ^{3} \theta\left(\alpha^{2} a_{2}-\beta b_{1}\right)-\alpha^{4} \beta^{5} b_{2}\left(\alpha^{2}+\beta\right)^{2} \sin ^{8} \theta, \\
& C_{2}=\sqrt{\alpha^{2} \beta^{2}\left(\beta\left(\alpha^{2}+\beta\right) \sin ^{2} \theta-\alpha^{2} \cos ^{2} \theta\left(\alpha^{2}+\beta R^{2}+\beta\right)\right)}\left(\alpha ^ { 2 } \beta ^ { 4 } \left(\alpha^{2}\right.\right. \\
& +\beta) \sin ^{7} \theta\left(\alpha^{2} b_{5}+\alpha a_{2}+\beta b_{5}\right)+\alpha^{6} \beta^{2}\left(\alpha^{2}+\beta\right) \cos ^{7} \theta\left(\alpha^{2} a_{3}+3 \alpha a_{1}\right. \\
& \left.+a_{3} \beta\right)-\alpha^{2} \beta^{3}\left(\alpha^{2}+\beta\right) \sin ^{6} \theta \cos \theta\left(\alpha^{4} a_{5}+\alpha^{2} a_{5} \beta-\alpha^{2} \beta b_{4}-\alpha a_{1} \beta\right. \\
& \left.-2 \alpha \beta b_{2}-\beta^{2} b_{4}\right)+\alpha^{4} \beta^{2}\left(\alpha^{2}+\beta\right) \sin ^{2} \theta \cos ^{5} \theta\left(\alpha^{4} a_{3}+\alpha^{4} a_{5}\right. \\
& \left.+3 \alpha^{3} a_{1}+\alpha^{2} a_{5} \beta-\alpha^{2} \beta b_{4}-4 \alpha a_{1} \beta-2 \alpha \beta b_{2}-a_{3} \beta^{2}-\beta^{2} b_{4}\right) \\
& +\alpha^{4} \beta^{2}\left(\alpha^{2}+\beta\right) \sin \theta \cos ^{6} \theta\left(\alpha^{4} a_{4}+3 \alpha^{3} a_{2}+\alpha^{2} a_{4} \beta-\alpha^{2} \beta b_{3}\right. \\
& \left.-2 \alpha \beta b_{1}-\beta^{2} b_{3}\right)-\alpha^{2} \beta^{3}\left(\alpha^{2}+\beta\right) \sin ^{5} \theta \cos ^{2} \theta\left(\alpha^{4} a_{4}+\alpha^{4} b_{5}\right. \\
& \left.+4 \alpha^{3} a_{2}+\alpha^{2} a_{4} \beta-\alpha^{2} \beta b_{3}-\alpha a_{2} \beta-2 \alpha \beta b_{1}-\beta^{2} b_{3}-\beta^{2} b_{5}\right) \\
& +\alpha^{2} \beta^{2}\left(\alpha^{2}+\beta\right) \sin ^{4} \theta \cos ^{3} \theta\left(\alpha^{6} a_{5}-\alpha^{4} a_{3} \beta-\alpha^{4} \beta b_{4}-4 \alpha^{3} a_{1} \beta\right. \\
& \left.-2 \alpha^{3} \beta b_{2}-\alpha^{2} a_{3} \beta^{2}-\alpha^{2} a_{5} \beta^{2}+\alpha a_{1} \beta^{2}+2 \alpha \beta^{2} b_{2}+\beta^{3} b_{4}\right) \\
& +\alpha^{2} \beta^{2}\left(\alpha^{2}+\beta\right) \sin ^{3} \theta \cos ^{4} \theta\left(\alpha^{6} a_{4}+3 \alpha^{5} a_{2}-\alpha^{4} \beta b_{3}-\alpha^{4} \beta b_{5}\right. \\
& \left.\left.-4 \alpha^{3} a_{2} \beta-2 \alpha^{3} \beta b_{1}-\alpha^{2} a_{4} \beta^{2}-\alpha^{2} \beta^{2} b_{5}+2 \alpha \beta^{2} b_{1}+\beta^{3} b_{3}\right)\right), \\
& C_{3}=\alpha^{6} \beta^{3}\left(\alpha^{2}+\beta\right)\left(2 a_{1} \alpha^{4}-a_{6} \beta \alpha^{4}-2 a_{3} \beta \alpha^{3}-2 a_{6} \beta^{2} \alpha^{2}-3 a_{1} \beta \alpha^{2}\right. \\
& \left.-2 a_{3} \beta^{2} \alpha-a_{6} \beta^{3}\right) \cos ^{8} \theta+\alpha^{4} \beta^{3}\left(\alpha^{2}+\beta\right)\left(2 a_{2} \alpha^{6}-a_{7} \beta \alpha^{6}\right. \\
& -2 a_{4} \beta \alpha^{5}-2 a_{7} \beta^{2} \alpha^{4}-3 a_{2} \beta \alpha^{4}-\beta b_{1} \alpha^{4}+\beta^{2} b_{6} \alpha^{4}-2 a_{4} \beta^{2} \alpha^{3} \\
& +\beta^{2} b_{3} \alpha^{3}-a_{7} \beta^{3} \alpha^{2}+\beta^{2} b_{1} \alpha^{2}+2 \beta^{3} b_{6} \alpha^{2}+\beta^{3} b_{3} \alpha \\
& \left.+\beta^{4} b_{6}\right) \sin \theta \cos ^{7} \theta+\alpha^{4} \beta^{3}\left(\alpha^{2}+\beta\right)\left(2 a_{1} \alpha^{6}-a_{6} \beta \alpha^{6}-a_{8} \beta \alpha^{6}\right. \\
& -2 a_{3} \beta \alpha^{5}-2 a_{5} \beta \alpha^{5}-a_{6} \beta^{2} \alpha^{4}-2 a_{8} \beta^{2} \alpha^{4}-6 a_{1} \beta \alpha^{4}-\beta b_{2} \alpha^{4} \\
& +\beta^{2} b_{7} \alpha^{4}+a_{3} \beta^{2} \alpha^{3}-2 a_{5} \beta^{2} \alpha^{3}+\beta^{2} b_{4} \alpha^{3}+a_{6} \beta^{3} \alpha^{2}-a_{8} \beta^{3} \alpha^{2} \\
& +5 a_{1} \beta^{2} \alpha^{2}+\beta^{2} b_{2} \alpha^{2}+2 \beta^{3} b_{7} \alpha^{2}+3 a_{3} \beta^{3} \alpha+\beta^{3} b_{4} \alpha+a_{6} \beta^{4} \\
& \left.+\beta^{4} b_{7}\right) \sin ^{2} \theta \cos ^{6} \theta+\alpha^{2} \beta^{3}\left(\alpha^{2}+\beta\right)\left(2 a_{2} \alpha^{8}-a_{7} \beta \alpha^{8}-a_{9} \beta \alpha^{8}\right. \\
& -2 a_{4} \beta \alpha^{7}-a_{7} \beta^{2} \alpha^{6}-2 a_{9} \beta^{2} \alpha^{6}-6 a_{2} \beta \alpha^{6}-\beta b_{1} \alpha^{6}+\beta^{2} b_{6} \alpha^{6} \\
& +\beta^{2} b_{8} \alpha^{6}+a_{4} \beta^{2} \alpha^{5}+\beta^{2} b_{3} \alpha^{5}+\beta^{2} b_{5} \alpha^{5}+a_{7} \beta^{3} \alpha^{4}-a_{9} \beta^{3} \alpha^{4} \\
& +5 a_{2} \beta^{2} \alpha^{4}+2 \beta^{2} b_{1} \alpha^{4}+\beta^{3} b_{6} \alpha^{4}+2 \beta^{3} b_{8} \alpha^{4}+3 a_{4} \beta^{3} \alpha^{3}+\beta^{3} b_{5} \alpha^{3} \\
& \left.+a_{7} \beta^{4} \alpha^{2}-\beta^{3} b_{1} \alpha^{2}-\beta^{4} b_{6} \alpha^{2}+\beta^{4} b_{8} \alpha^{2}-\beta^{4} b_{3} \alpha-\beta^{5} b_{6}\right) \sin ^{3} \theta \cos ^{5} \theta \\
& -\alpha^{2} \beta^{4}\left(\alpha^{2}+\beta\right)\left(a_{8} \alpha^{8}+2 a_{5} \alpha^{7}+3 a_{1} \alpha^{6}-a_{6} \beta \alpha^{6}+a_{8} \beta \alpha^{6}+b_{2} \alpha^{6}\right. \\
& -\beta b_{7} \alpha^{6}-\beta b_{9} \alpha^{6}-3 a_{3} \beta \alpha^{5}-a_{5} \beta \alpha^{5}-\beta b_{4} \alpha^{5}-2 a_{6} \beta^{2} \alpha^{4}-a_{8} \beta^{2} \alpha^{4} \\
& -6 a_{1} \beta \alpha^{4}-2 \beta b_{2} \alpha^{4}-\beta^{2} b_{7} \alpha^{4}-2 \beta^{2} b_{9} \alpha^{4}-2 a_{3} \beta^{2} \alpha^{3}-3 a_{5} \beta^{2} \alpha^{3} \\
& -a_{6} \beta^{3} \alpha^{2}-a_{8} \beta^{3} \alpha^{2}+2 a_{1} \beta^{2} \alpha^{2}+\beta^{2} b_{2} \alpha^{2}+\beta^{3} b_{7} \alpha^{2}-\beta^{3} b_{9} \alpha^{2}+a_{3} \beta^{3} \alpha \\
& \left.+\beta^{3} b_{4} \alpha+\beta^{4} b_{7}\right) \sin ^{4} \theta \cos ^{4} \theta-\alpha^{2} \beta^{4}\left(\alpha^{2}+\beta\right)\left(a_{9} \alpha^{8}+3 a_{2} \alpha^{6}-a_{7} \beta \alpha^{6}\right. \\
& +a_{9} \beta \alpha^{6}-\beta b_{8} \alpha^{6}-3 a_{4} \beta \alpha^{5}-\beta b_{5} \alpha^{5}-2 a_{7} \beta^{2} \alpha^{4}-a_{9} \beta^{2} \alpha^{4}-6 a_{2} \beta \alpha^{4} \\
& -\beta b_{1} \alpha^{4}+\beta^{2} b_{6} \alpha^{4}-\beta^{2} b_{8} \alpha^{4}-2 a_{4} \beta^{2} \alpha^{3}+\beta^{2} b_{3} \alpha^{3}-a_{7} \beta^{3} \alpha^{2}-a_{9} \beta^{3} \alpha^{2}
\end{aligned}
$$




$$
\begin{aligned}
& +2 a_{2} \beta^{2} \alpha^{2}+\beta^{2} b_{1} \alpha^{2}+2 \beta^{3} b_{6} \alpha^{2}+\beta^{3} b_{8} \alpha^{2}+a_{4} \beta^{3} \alpha+\beta^{3} b_{3} \alpha+\beta^{3} b_{5} \alpha \\
& \left.+\beta^{4} b_{6}+\beta^{4} b_{8}\right) \sin ^{5} \theta \cos ^{3} \theta+\alpha^{2} \beta^{5}\left(\alpha^{2}+\beta\right)\left(a_{8} \alpha^{6}+b_{9} \alpha^{6}+3 a_{5} \alpha^{5}\right. \\
& +a_{1} \alpha^{4}+2 a_{8} \beta \alpha^{4}+b_{2} \alpha^{4}-\beta b_{7} \alpha^{4}+\beta b_{9} \alpha^{4}-a_{3} \beta \alpha^{3}+2 a_{5} \beta \alpha^{3} \\
& -\beta b_{4} \alpha^{3}+a_{8} \beta^{2} \alpha^{2}-2 a_{1} \beta \alpha^{2}-\beta b_{2} \alpha^{2}-2 \beta^{2} b_{7} \alpha^{2}-\beta^{2} b_{9} \alpha^{2}-a_{3} \beta^{2} \alpha \\
& \left.-a_{5} \beta^{2} \alpha-\beta^{2} b_{4} \alpha-\beta^{3} b_{7}-\beta^{3} b_{9}\right) \sin ^{6} \theta \cos ^{2} \theta+\alpha^{2} \beta^{5}\left(\alpha^{2}+\beta\right)\left(a_{9} \alpha^{6}\right. \\
& +a_{2} \alpha^{4}+2 a_{9} \beta \alpha^{4}-\beta b_{8} \alpha^{4}-a_{4} \beta \alpha^{3}-\beta b_{5} \alpha^{3}+a_{9} \beta^{2} \alpha^{2}-2 a_{2} \beta \alpha^{2} \\
& \left.-2 \beta^{2} b_{8} \alpha^{2}-a_{4} \beta^{2} \alpha-\beta^{2} b_{5} \alpha-\beta^{3} b_{8}\right) \sin ^{7} \theta \cos \theta \\
& -\alpha^{2} \beta^{6}\left(\alpha^{2}+\beta\right)^{2}\left(b_{9} \alpha^{2}+a_{5} \alpha+\beta b_{9}\right) \sin ^{8} \theta, \\
& C_{4}=\sqrt{\alpha^{2} \beta^{2}\left(\beta\left(\alpha^{2}+\beta\right) \sin ^{2} \theta-\alpha^{2} \cos ^{2} \theta\left(\alpha^{2}+\beta R^{2}+\beta\right)\right)}\left(\alpha a _ { 9 } \beta ^ { 5 } \left(\alpha^{2}\right.\right. \\
& +\beta)^{2} \sin ^{7} \theta-\alpha \beta^{4}\left(\alpha^{2}+\beta\right) \sin ^{6} \theta \cos \theta\left(\alpha^{3} a_{5}-\alpha^{2} a_{8} \beta-\alpha a_{5} \beta-a_{8} \beta^{2}\right) \\
& -\alpha \beta^{4} \sin ^{5} \theta \cos ^{2} \theta\left(\alpha^{6} a_{9}+\alpha^{5} a_{4}+3 \alpha^{4} a_{2}-\alpha^{4} a_{7} \beta+\alpha^{4} a_{9} \beta-\alpha^{2} a_{2} \beta\right. \\
& \left.-2 \alpha^{2} a_{7} \beta^{2}-\alpha^{2} a_{9} \beta^{2}-\alpha a_{4} \beta^{2}-a_{7} \beta^{3}-a_{9} \beta^{3}\right)+\alpha^{3} \beta^{3} \cos ^{7} \theta\left(\alpha^{5} a_{3}\right. \\
& \left.+3 \alpha^{4} a_{1}-\alpha^{4} a_{6} \beta-\alpha^{2} a_{1} \beta-2 \alpha^{2} a_{6} \beta^{2}-\alpha a_{3} \beta^{2}-a_{6} \beta^{3}\right) \\
& +\alpha^{3} \beta^{3} \sin \theta \cos ^{6} \theta\left(\alpha^{5} a_{4}+3 \alpha^{4} a_{2}-\alpha^{4} a_{7} \beta-\alpha^{2} a_{2} \beta-2 \alpha^{2} a_{7} \beta^{2}\right. \\
& \left.-\alpha a_{4} \beta^{2}-a_{7} \beta^{3}\right)+\alpha \beta^{3} \sin ^{2} \theta \cos ^{5} \theta\left(\alpha^{7} a_{3}+\alpha^{7} a_{5}+3 \alpha^{6} a_{1}\right. \\
& -\alpha^{6} a_{6} \beta-\alpha^{6} a_{8} \beta-\alpha^{5} a_{3} \beta-4 \alpha^{4} a_{1} \beta-\alpha^{4} a_{6} \beta^{2}-2 \alpha^{4} a_{8} \beta^{2}-\alpha^{3} a_{3} \beta^{2} \\
& \left.-\alpha^{3} a_{5} \beta^{2}+\alpha^{2} a_{1} \beta^{2}+\alpha^{2} a_{6} \beta^{3}-\alpha^{2} a_{8} \beta^{3}+\alpha a_{3} \beta^{3}+a_{6} \beta^{4}\right) \\
& +\alpha \beta^{3} \sin ^{4} \theta \cos ^{3} \theta\left(\alpha^{7} a_{5}-\alpha^{6} a_{8} \beta-\alpha^{5} a_{3} \beta-\alpha^{5} a_{5} \beta-3 \alpha^{4} a_{1} \beta\right. \\
& +\alpha^{4} a_{6} \beta^{2}-\alpha^{4} a_{8} \beta^{2}-\alpha^{3} a_{5} \beta^{2}+\alpha^{2} a_{1} \beta^{2}+2 \alpha^{2} a_{6} \beta^{3}+\alpha^{2} a_{8} \beta^{3}+\alpha a_{3} \beta^{3} \\
& \left.+\alpha a_{5} \beta^{3}+a_{6} \beta^{4}+a_{8} \beta^{4}\right)+\alpha \beta^{3} \sin ^{3} \theta \cos ^{4} \theta\left(\alpha^{7} a_{4}+3 \alpha^{6} a_{2}-\alpha^{6} a_{7} \beta\right. \\
& -\alpha^{6} a_{9} \beta-\alpha^{5} a_{4} \beta-4 \alpha^{4} a_{2} \beta-\alpha^{4} a_{7} \beta^{2}-2 \alpha^{4} a_{9} \beta^{2}-\alpha^{3} a_{4} \beta^{2}+\alpha^{2} a_{2} \beta^{2} \\
& \left.\left.+\alpha^{2} a_{7} \beta^{3}-\alpha^{2} a_{9} \beta^{3}+\alpha a_{4} \beta^{3}+a_{7} \beta^{4}\right)\right), \\
& C_{5}=\alpha^{4} \beta^{6}\left(\alpha^{2}+\beta\right) \sin ^{6} \theta \cos ^{2} \theta\left(\alpha^{2} a_{8}+2 \alpha a_{5}+a_{8} \beta\right)+\alpha^{4} a_{9} \beta^{6}\left(\alpha^{2}\right. \\
& +\beta)^{2} \sin ^{7} \theta \cos \theta+\alpha^{4} \beta^{4} \sin ^{3} \theta \cos ^{5} \theta\left(\alpha^{6} a_{2}-\alpha^{6} a_{7} \beta-\alpha^{6} a_{9} \beta\right. \\
& -2 \alpha^{5} a_{4} \beta-4 \alpha^{4} a_{2} \beta-\alpha^{4} a_{7} \beta^{2}-2 \alpha^{4} a_{9} \beta^{2}+3 \alpha^{2} a_{2} \beta^{2}+\alpha^{2} a_{7} \beta^{3} \\
& \left.-\alpha^{2} a_{9} \beta^{3}+2 \alpha a_{4} \beta^{3}+a_{7} \beta^{4}\right)+\alpha^{6} \beta^{4} \cos ^{8} \theta\left(\alpha^{4} a_{1}-\alpha^{4} a_{6} \beta-2 \alpha^{3} a_{3} \beta\right. \\
& \left.-3 \alpha^{2} a_{1} \beta-2 \alpha^{2} a_{6} \beta^{2}-2 \alpha a_{3} \beta^{2}-a_{6} \beta^{3}\right)-\alpha^{4} \beta^{5} \sin ^{5} \theta \cos ^{3} \theta\left(\alpha^{6} a_{9}\right. \\
& +\alpha^{4} a_{2}-\alpha^{4} a_{7} \beta+\alpha^{4} a_{9} \beta-2 \alpha^{3} a_{4} \beta-3 \alpha^{2} a_{2} \beta-2 \alpha^{2} a_{7} \beta^{2}-\alpha^{2} a_{9} \beta^{2} \\
& \left.-2 \alpha a_{4} \beta^{2}-a_{7} \beta^{3}-a_{9} \beta^{3}\right)+\alpha^{6} \beta^{4} \sin \theta \cos ^{7} \theta\left(\alpha^{4} a_{2}-\alpha^{4} a_{7} \beta\right. \\
& \left.-2 \alpha^{3} a_{4} \beta-3 \alpha^{2} a_{2} \beta-2 \alpha^{2} a_{7} \beta^{2}-2 \alpha a_{4} \beta^{2}-a_{7} \beta^{3}\right) \\
& -\alpha^{4} \beta^{5} \sin ^{4} \theta \cos ^{4} \theta\left(\alpha^{6} a_{8}+2 \alpha^{5} a_{5}+\alpha^{4} a_{1}-\alpha^{4} a_{6} \beta+\alpha^{4} a_{8} \beta\right. \\
& -2 \alpha^{3} a_{3} \beta-3 \alpha^{2} a_{1} \beta-2 \alpha^{2} a_{6} \beta^{2}-\alpha^{2} a_{8} \beta^{2}-2 \alpha a_{3} \beta^{2}-2 \alpha a_{5} \beta^{2} \\
& \left.-a_{6} \beta^{3}-a_{8} \beta^{3}\right)+\alpha^{4} \beta^{4} \sin ^{2} \theta \cos ^{6} \theta\left(\alpha^{6} a_{1}-\alpha^{6} a_{6} \beta-\alpha^{6} a_{8} \beta\right. \\
& -2 \alpha^{5} a_{3} \beta-2 \alpha^{5} a_{5} \beta-4 \alpha^{4} a_{1} \beta-\alpha^{4} a_{6} \beta^{2}-2 \alpha^{4} a_{8} \beta^{2}-2 \alpha^{3} a_{5} \beta^{2}
\end{aligned}
$$




$$
\begin{gathered}
\left.+3 \alpha^{2} a_{1} \beta^{2}+\alpha^{2} a_{6} \beta^{3}-\alpha^{2} a_{8} \beta^{3}+2 \alpha a_{3} \beta^{3}+a_{6} \beta^{4}\right), \\
Q_{4}=2\left(\alpha^{2}+\beta\right)^{2}\left(\alpha^{2} \cos ^{2} \theta-\beta \sin ^{2} \theta\right)^{3} S\left(S-\alpha \beta^{2} R \cos \theta\right),
\end{gathered}
$$

with

$$
S=\sqrt{\alpha^{2} \beta^{2}\left(\beta\left(\alpha^{2}+\beta\right) \sin ^{2} \theta-\alpha^{2} \cos ^{2} \theta\left(\alpha^{2}+\beta R^{2}+\beta\right)\right)} .
$$

Integrating the right part of differential equation (16) we obtain the averaging function $f:\left(0, \sqrt{-\alpha^{2} / \beta}\right) \rightarrow \mathbb{R}$ given by

$$
\begin{aligned}
f(R)= & \beta f_{0}\left[\frac{\left(3 \alpha^{4} a_{8}-3 \alpha^{4} b_{9}+\alpha^{2} a_{1}+\alpha^{2} a_{6} \beta+3 \alpha^{2} a_{8} \beta-\alpha^{2} \beta b_{7}\right.}{4 \alpha(-\beta)^{3 / 2}\left(\alpha^{2}+\beta\right)}\right. \\
& \left.+\frac{\left.-3 \alpha^{2} \beta b_{9}+\alpha^{2} b_{2}+a_{6} \beta^{2}-\beta^{2} b_{7}\right)}{4 \alpha(-\beta)^{3 / 2}\left(\alpha^{2}+\beta\right)}\right] \\
& +\beta^{2} f_{1}\left[\frac{\left(\alpha^{6} a_{8}+\alpha^{5} a_{5}+\alpha^{4} a_{1}-\alpha^{4} a_{6} \beta+2 \alpha^{4} a_{8} \beta-\alpha^{3} a_{3} \beta\right.}{4 \alpha^{3}(-\beta)^{3 / 2}\left(\alpha^{2}+\beta\right)^{2}}\right. \\
& \left.+\frac{\left.\alpha^{3} a_{5} \beta-\alpha^{2} a_{1} \beta-2 \alpha^{2} a_{6} \beta^{2}+\alpha^{2} a_{8} \beta^{2}-\alpha a_{3} \beta^{2}-a_{6} \beta^{3}\right)}{4 \alpha^{3}(-\beta)^{3 / 2}\left(\alpha^{2}+\beta\right)^{2}}\right] \\
& -f_{2} \frac{\beta\left(\alpha^{2}+\beta\right)^{2}\left(a_{8}-b_{9}\right)}{2(-\beta)^{3 / 2}}+f_{3} \frac{\left(\alpha^{2} a_{8}-\alpha^{2} b_{9}+a_{6} \beta-\beta b_{7}\right)}{2(-\beta)^{3 / 2}},
\end{aligned}
$$

where

$$
\begin{gathered}
f_{0}=R, \quad, f_{1}=R^{3}, \quad f_{2}=R \sqrt{\alpha^{2}+\beta R^{2}}, \\
f_{3}=\left(\alpha-\left(\alpha^{2}+\beta\right)^{2} \sqrt{\alpha^{2}+\beta R^{2}}\right) .
\end{gathered}
$$

The Wronskians $W\left(f_{0}, \ldots, f_{k}\right)$, for $k=0, . ., 3$ are given by

$$
\begin{aligned}
W\left(f_{0}\right)= & R, \quad W\left(f_{0}, f_{1}\right)=2 R^{3}, \quad W\left(f_{0}, f_{1}, f_{2}\right)=-\frac{2 \beta^{2} R^{6}}{\left(\alpha^{2}+\beta R^{2}\right)^{3 / 2}}, \\
W\left(f_{0}, \ldots, f_{3}\right)= & -\frac{12 \beta^{2} R^{2}}{\left(\alpha^{2}+\beta R^{2}\right)^{7 / 2}}\left(( \alpha ^ { 2 } + \beta ) ^ { 2 } \sqrt { \alpha ^ { 2 } + \beta R ^ { 2 } } \left(8 \alpha^{4}+8 \alpha^{2} \beta R^{2}\right.\right. \\
& \left.\left.+\beta^{2} R^{4}\right)-4 \alpha\left(\alpha^{2}+\beta R^{2}\right)\left(2 \alpha^{2}+\beta R^{2}\right)\right) .
\end{aligned}
$$

Obviously the first three Wronskians do not vanish because $R \in\left(0, \sqrt{-\alpha^{2} / \beta}\right)$. The last Wronskian is equal to zero if and only if

$\left(\alpha^{2}+\beta\right)^{2} \sqrt{\alpha^{2}+\beta R^{2}}\left(8 \alpha^{4}+8 \alpha^{2} \beta R^{2}+\beta^{2} R^{4}\right)-4 \alpha\left(\alpha^{2}+\beta R^{2}\right)\left(2 \alpha^{2}+\beta R^{2}\right)=0$.

Passing the term $\left[\left(\alpha^{2}+\beta\right)^{2} \sqrt{\alpha^{2}+\beta R^{2}}\left(8 \alpha^{4}+8 \alpha^{2} \beta R^{2}+\beta^{2} R^{4}\right)\right.$ to the right hand side of the previous equality and taking the square in both sides we 
get

$$
\begin{aligned}
& \left(-\alpha^{2}-\beta R^{2}\right)\left(64 \alpha^{16}+128 \alpha^{14} \beta R^{2}+256 \alpha^{14} \beta+80 \alpha^{12} \beta^{2} R^{4}\right. \\
& +512 \alpha^{12} \beta^{2} R^{2}+384 \alpha^{12} \beta^{2}+16 \alpha^{10} \beta^{3} R^{6}+320 \alpha^{10} \beta^{3} R^{4}+768 \alpha^{10} \beta^{3} R^{2} \\
& +256 \alpha^{10} \beta^{3}+\alpha^{8} \beta^{4} R^{8}+64 \alpha^{8} \beta^{4} R^{6}+480 \alpha^{8} \beta^{4} R^{4}+512 \alpha^{8} \beta^{4} R^{2}+64 \alpha^{8} \beta^{4} \\
& -64 \alpha^{8}+4 \alpha^{6} \beta^{5} R^{8}+96 \alpha^{6} \beta^{5} R^{6}+320 \alpha^{6} \beta^{5} R^{4}+128 \alpha^{6} \beta^{5} R^{2}-128 \alpha^{6} \beta R^{2} \\
& +6 \alpha^{4} \beta^{6} R^{8}+64 \alpha^{4} \beta^{6} R^{6}+80 \alpha^{4} \beta^{6} R^{4}-80 \alpha^{4} \beta^{2} R^{4}+4 \alpha^{2} \beta^{7} R^{8}+16 \alpha^{2} \beta^{7} R^{6} \\
& \left.-16 \alpha^{2} \beta^{3} R^{6}+\beta^{8} R^{8}\right)=0 .
\end{aligned}
$$

The factor $\left(-\alpha^{2}-\beta R^{2}\right)$ does not vanish. Taking the variable change $h=$ $R^{2}$, in the second factor in the left hand side of the previous equation we obtain the polynomial of degree 4

$$
\begin{aligned}
p(h)= & 64 \alpha^{16}+128 \alpha^{14} \beta h+256 \alpha^{14} \beta+80 \alpha^{12} \beta^{2} h^{2}+512 \alpha^{12} \beta^{2} h \\
& +384 \alpha^{12} \beta^{2}+16 \alpha^{10} \beta^{3} h^{3}+320 \alpha^{10} \beta^{3} h^{2}+768 \alpha^{10} \beta^{3} h+256 \alpha^{10} \beta^{3} \\
& +64 \alpha^{8} \beta^{4} h^{3}+480 \alpha^{8} \beta^{4} h^{2}+512 \alpha^{8}+\alpha^{8} \beta^{4} h^{4} \beta^{4} h+64 \alpha^{8} \beta^{4} \\
& -64 \alpha^{8}+4 \alpha^{6} \beta^{5} h^{4}+96 \alpha^{6} \beta^{5} h^{3}+320 \alpha^{6} \beta^{5} h^{2}+128 \alpha^{6} \beta^{5} h \\
& -128 \alpha^{6} \beta h+6 \alpha^{4} \beta^{6} h^{4}+64 \alpha^{4} \beta^{6} h^{3}+80 \alpha^{4} \beta^{6} h^{2}-80 \alpha^{4} \beta^{2} h^{2} \\
& +4 \alpha^{2} \beta^{7} h^{4}+16 \alpha^{2} \beta^{7} h^{3}-16 \alpha^{2} \beta^{3} h^{3}+\beta^{8} h^{4} .
\end{aligned}
$$

For get its zeros we apply the result given in [17]. We rewrite this polynomial as follows

$$
p(h)=s_{4}+s_{3} h+s_{2} h^{2}+s_{1} h^{3}+s_{0} h^{4} .
$$

We look to the expressions

$$
\begin{aligned}
D_{2}= & 3 s_{1}^{2}-8 s_{0} s_{2} \\
D_{3}= & 16 s_{0}^{2} s_{2} s_{4}-18 s_{0}^{2} s_{3}^{2}-6 s_{0} s_{1}^{2} s_{4}+14 s_{0} s_{1} s_{2} s_{3}-4 s_{0} s_{2}^{3}-3 s_{1}^{3} s_{3}+s_{1}^{2} s_{2}^{2} \\
D_{4}= & 256 s_{0}^{3} s_{4}^{3}-192 s_{0}^{2} s_{1} s_{3} s_{4}^{2}-128 s_{0}^{2} s_{2}^{2} s_{4}^{2}+144 s_{0}^{2} s_{2} s_{3}^{2} s_{4}-27 s_{0}^{2} s_{3}^{4} \\
& +144 s_{0} s_{1}^{2} s_{2} s_{4}^{2}-6 s_{0} s_{1}^{2} s_{3}^{2} s_{4}-80 s_{0} s_{1} s_{2}^{2} s_{3} s_{4}+18 s_{0} s_{1} s_{2} s_{3}^{3}+16 s_{0} s_{2}^{4} s_{4} \\
& -4 s_{0} s_{2}^{3} s_{3}^{2}-27 s_{1}^{4} s_{4}^{2}+18 s_{1}^{3} s_{2} s_{3} s_{4}-4 s_{1}^{3} s_{3}^{3}-4 s_{1}^{2} s_{2}^{3} s_{4}+s_{1}^{2} s_{2}^{2} s_{3}^{2}, \\
E_{1}= & 8 s_{0}^{2} s_{3}-4 s_{0} s_{1} s_{2}+s_{1}^{3} .
\end{aligned}
$$

Thus we get

$$
\begin{aligned}
D_{2}= & 128 \alpha^{4} \beta^{6}\left(\alpha^{2}+\beta-1\right)\left(\alpha^{2}+\beta+1\right)\left(\left(\alpha^{2}+\beta\right)^{2}+1\right)\left(\left(\alpha^{2}+\beta\right)^{4}-6\right), \\
D_{3}= & 16384 \alpha^{12} \beta^{10}\left(\alpha^{2}+\beta-1\right)^{2}\left(\alpha^{2}+\beta+1\right)^{2}\left(\left(\alpha^{2}+\beta\right)^{2}+1\right)^{2}\left(17\left(\alpha^{2}+\beta\right)^{4}\right. \\
& -4), \\
D_{4}= & 67108864 \alpha^{24} \beta^{12}\left(\alpha^{2}+\beta-1\right)^{3}\left(\alpha^{2}+\beta\right)^{4}\left(\alpha^{2}+\beta+1\right)^{3}\left(\left(\alpha^{2}+\beta\right)^{2}+1\right)^{3}, \\
E_{1}= & -1024 \alpha^{6} \beta^{9}\left(\alpha^{2}+\beta-1\right)\left(\alpha^{2}+\beta+1\right)\left(\left(\alpha^{2}+\beta\right)^{2}+1\right)\left(3\left(\alpha^{2}+\beta\right)^{4}-4\right) .
\end{aligned}
$$

We recall that $\beta<0, \alpha^{2}+\beta<0$ and $R \in\left(0, \sqrt{-\alpha^{2} / \beta}\right)$. Thus,

(i) If $D_{4}=0$, then $D_{2}=0$ and $D_{3}=0$. This implies that the polynomial $p$ has 1 real root of multiplicity 4 (see [17]). However $D_{4}=0$ if 
and only if $\alpha^{2}+\beta+1=0$. We have that

$$
p(0)=64 \alpha^{8}\left(\alpha^{2}+\beta-1\right)\left(\alpha^{2}+\beta+1\right)\left(\left(\alpha^{2}+\beta\right)^{2}+1\right) .
$$

Since $\alpha^{2}+\beta+1=0, p(0)=0$. Therefore $h=0$ is the real root of $p$ of multiplicity 4 . This is impossible because $R \in\left(0, \sqrt{-\alpha^{2} / \beta}\right)$.

(ii) If $D_{4}>0$, then $\alpha^{2}+\beta+1<0$. Thus $D_{2}<0$ and the polynomial $p$ does not have real roots (see [17]).

(iii) If $D_{4}<0$, i.e., $-1<\alpha^{2}+\beta<0$, then the polynomial $p$ has 2 real roots but this can not happens because $\alpha^{2}+\beta \notin(-1,0)$.

Hence the Wronskian $W\left(f_{0}, . ., f_{3}\right)$ does not vanish for $R \in\left(0, \sqrt{-\alpha^{2} / \beta}\right)$. Therefore, since that $\left(f_{0}, \ldots, f_{3}\right)$ is an ECT-system the averaging function $f$ has at most 3 simple zeros and they are reached. By Theorem 4, these zeros provide 3 limit cycles for system (2).

If we suppose that $\alpha^{2}+\beta>0$ then we repeat the calculus with the positive function

$$
\bar{\rho}=\frac{\beta^{2} R\left(\alpha^{2}+\beta\right)}{\sqrt{\alpha^{2} \beta^{2}\left(\beta\left(\alpha^{2}+\beta\right) \sin ^{2} \theta-\alpha^{2} \cos ^{2} \theta\left(\alpha^{2}+\beta R^{2}+\beta\right)\right)}+\alpha \beta^{2} R \cos \theta}
$$

and we ensure that system (2) has at most 3 limit cycles and they are reached since $\alpha^{2}+\beta \notin(0,1)$. So Theorem 2 is proved.

\section{Comments about the Classes $P_{3}$ And $P_{5}$}

The computation of the number $\mathcal{N}$ for the classes $P_{3}$ and $P_{5}$ remains open. For the class $P_{3}$, after translating the center of the system to the origin of coordinates, the new differential system has the first integral

$$
H(x, y)=\frac{\alpha^{2} x^{2}-2 \alpha^{2} x y+\alpha^{2} y^{2}+x^{2}+2 x y+y^{2}}{4 \alpha^{2}\left(\alpha^{4}-2 \alpha^{2} x-2 \alpha^{2} y+\alpha^{2}+x^{2}+2 x y+y^{2}\right)},
$$

and the corresponding integrating factor is

$$
\mu(x, y)=\frac{1}{\left(\left(-\alpha^{2}+x+y\right)^{2}+\alpha^{2}\right)^{2}} .
$$

Repeating the same process than in the proofs of Theorems 1 and 2, we find a positive function $\rho$ given by

$$
\rho=\frac{4 R^{2} \alpha^{4}(\sin \theta+\cos \theta)-2 R \sqrt{S}}{2 \sin \theta \cos \theta\left(\left(4 R^{2}+1\right) \alpha^{2}-1\right)+\left(4 R^{2}-1\right) \alpha^{2}-1},
$$

where

$$
S=\alpha^{4}\left(\left(2-4 R^{2}\right) \alpha^{2}-2 \sin \theta \cos \theta\left(4 R^{2} \alpha^{2}+\alpha^{4}-1\right)+\alpha^{4}+1\right),
$$

and we obtain a system in the standard form of the averaging theorem

$$
\frac{d R}{d \varphi}=\varepsilon \sum_{i=1}^{11} \frac{D_{i}(\varphi, \alpha, \beta, a, b)}{Q_{5}(R, \varphi, \alpha, \beta)} R^{i}+\mathcal{O}\left(\varepsilon^{2}\right),
$$


where the denominator $Q_{5}$ is given by

$$
\begin{aligned}
Q_{5}= & \alpha^{4}\left(\left(\alpha^{2}-1\right) \sin 2 \theta-\alpha^{2}-1\right)^{3}\left(\sin 2 \theta\left(\left(4 R^{2}+1\right) \alpha^{2}-1\right)+\left(4 R^{2}-1\right) \alpha^{2}\right. \\
& -1)\left(\left(\alpha^{2}-1\right) \sin 2 \theta\left(\left(4 R^{2}-1\right) \alpha^{2}-1\right)+\alpha^{2}\left(4 R^{2}\left(\alpha^{2}-1\right)+\alpha^{2}+2\right)\right. \\
& -4 R(\sin \theta \\
& +\cos \theta \sqrt{\alpha^{4}\left(\alpha^{2}\left(-4 R^{2}+\alpha^{2}+2\right)-\sin 2 \theta\left(4 R^{2} \alpha^{2}+\alpha^{4}-1\right)+1\right)} \\
& +1)^{2}\left(\sin 2 \theta\left(4 R^{2} \alpha^{2}+\alpha^{4}-1\right)+2 R \sin \theta\right) \\
& +\cos \theta) \sqrt{\alpha^{4}\left(\alpha^{2}\left(-4 R^{2}+\alpha^{2}+2\right)-\sin 2 \theta\left(4 R^{2} \alpha^{2}+\alpha^{4}-1\right)+1\right)} \\
& +\left(2 R \alpha-\alpha^{2}-1\right)\left(2 R \alpha+\alpha^{2}+1\right) .
\end{aligned}
$$

Due to the expression of the denominator $Q_{5}$ we cannot compute the integral for applying the averaging theory of first order for equation (17). The same problem with the integral happens with the class $P_{5}$. However we obtain the number of infinitesimal limit cycles that bifurcate from origin for these classes by using the averaging of fifth order.

\section{Proof of Theorem 3}

6.1. Proof for the Class $P_{3}$. In order to simplify the computations we denote $1+\alpha^{2}$ by $a$ in the system corresponding to the class $P_{3}$. Translating the center $(-a / 2,-a / 2)$ of the class $P_{3}$ to the origin of coordinates we obtain the polynomial differential system

$$
\begin{aligned}
\dot{X} & =P(X, Y)=\frac{1}{2}(a-2 X)\left(X(a-X-2)-a Y+Y^{2}\right), \\
\dot{Y} & =Q(X, Y)=-\frac{1}{2}(a-2 Y)\left(a(Y-X)+X^{2}-Y(Y+2)\right) .
\end{aligned}
$$

We perturb the previous system up to fifth order as follows

$$
\begin{aligned}
\dot{X} & =P(X, Y)+\varepsilon^{i} \sum_{i=1}^{5} p_{i}(X, Y), \\
\dot{Y} & =Q(X, Y)+\varepsilon^{i} \sum_{i=1}^{5} q_{i}(X, Y),
\end{aligned}
$$

where

$$
\begin{aligned}
p_{1}(X, Y)= & a_{1} X+a_{2} Y+a_{3} X^{2}+a_{4} X Y+a_{5} Y^{2}+a_{6} X^{3}+a_{7} X^{2} Y \\
& +a_{8} X Y^{2}+a_{9} Y^{3} \\
q_{1}(X, Y)= & \alpha_{1} X+\alpha_{2} Y+\alpha_{3} X^{2}+\alpha_{4} X Y+\alpha_{5} Y^{2}+\alpha_{6} X^{3}+\alpha_{7} X^{2} Y \\
& +\alpha_{8} X Y^{2}+\alpha_{9} Y^{3}
\end{aligned}
$$

The polynomials $p_{2}, p_{3}, p_{4}$ and $p_{5}$ are given changing the character $a$ by $b, c, d$ and $e$ in $p_{1}$ respectively and the polynomials $q_{2}, q_{3}, q_{4}$ and $q_{5}$ are obtained changing the character $\alpha$ by $\beta, \gamma, \delta$ and $\phi$ respectively.

We take the variable changes $X=\varepsilon x$ and $Y=\varepsilon y$ just to study the bifurcations surround the origin and we pass the system obtained in the 
variables $x$ and $y$ to the real Jordan Form. Due the size of the system obtained in the real Jordan form we do not give it explicitly here. After we pass the system to the polar coordinates taking $x=r \cos \theta$ and $y=r \sin \theta$ and we take the quotient $\dot{r} / \dot{\theta}$ and we obtain the differential equation in the standard form of the averaging of fifth order

$$
\frac{d r}{d \theta}=\sum_{i=1}^{5} K_{i} \varepsilon_{i} .
$$

The coefficients $K_{i}$ are very large and we do not explicitly it here. Calculating the functions $f_{i}$ as in Theorem 4 we obtain

$$
f_{1}=\frac{\pi r\left(a_{1}+\alpha_{2}\right)}{\sqrt{(a-1) a^{2}}} .
$$

Take $\alpha_{2}=-a_{1}$. So we have $f_{1}=0$ and we obtain following the process in Theorem 4

$$
f_{2}=\frac{\pi r\left(b_{1}+\beta_{2}\right)}{\sqrt{(a-1) a^{2}}} .
$$

Take $b_{1}=-\beta_{2}$. Thus $f_{2}=0$ and we get

$$
\begin{aligned}
f_{3}= & \frac{\pi(a-1) a^{2} r\left(c_{1}+\gamma_{2}\right)}{\left((a-1) a^{2}\right)^{3 / 2}}+\frac{\pi(a-1) r^{3}}{4 a\left((a-1) a^{2}\right)^{3 / 2}}\left(3 a^{3} a_{6}+2 a^{3} a_{7}+a^{3} \alpha_{7}\right. \\
& +a^{3} a_{8}+2 a^{3} \alpha_{8}+3 a^{3} \alpha_{9}+4 a^{2} a_{3}+4 a^{2} \alpha_{3}+4 a^{2} a_{4}+4 a^{2} \alpha_{4}+4 a^{2} a_{5} \\
& +4 a^{2} \alpha_{5}-4 a^{2} a_{7}-4 a^{2} \alpha_{8}+8 a \alpha_{1}+8 a a_{2}-8 a a_{4}-8 a \alpha_{4}-16 \alpha_{1} \\
& \left.-16 a_{2}\right) .
\end{aligned}
$$

Solving in the variable $a_{4}$ the equation obtained equalling to zero the factor that multiplies $\frac{\pi(a-1) r^{3}}{4 a\left((a-1) a^{2}\right)^{3 / 2}}$ and taking $c_{1}=-\gamma_{2}$ we have $f_{3}=0$. Following the process we obtain

$$
f_{4}=A_{1} r+A_{3} r^{3}
$$

where

$$
A_{1}=\frac{\pi r\left(d_{1}+\delta_{2}\right)}{\sqrt{(a-1) a^{2}}} .
$$

The coefficient $A_{3}$ is too large thus we do not explicitly it here but it is given in the variables

$$
\begin{aligned}
& a, a_{1}, a_{2}, a_{3}, a_{5}, a_{6}, a_{7}, a_{8}, b_{2}, b_{3}, b_{4}, b_{5}, b_{6}, b_{7}, b_{8}, \alpha_{1}, \alpha_{3}, \alpha_{4}, \alpha_{5}, \alpha_{7}, \alpha_{8}, \alpha_{9}, \\
& \beta_{1}, \beta_{3}, \beta_{4}, \beta_{5}, \beta_{7}, \beta_{8}, \beta_{9} .
\end{aligned}
$$

The coefficients $A_{1}$ and $A_{3}$ are linearly independent because the parameter $d_{1}$ that appears in $A_{1}$ does not appear in $A_{3}$. Taking $d_{1}=-\delta_{2}$ and solving $A_{3}=0$ in the variable $\beta_{3}$ we have that $f_{4}=0$. Following the process we obtain

$$
f_{5}=C_{1} r+C_{3} r^{3}+C_{5} r^{5},
$$


where

$$
C_{1}=-\frac{\pi r\left(e_{1}+\phi_{2}\right)}{\sqrt{(a-1) a^{2}}} .
$$

Again we do not have explicitly $C_{3}$ but it is given in the variables

$$
\begin{aligned}
& a, a_{1}, a_{2}, a_{3}, a_{5}, a_{6}, a_{7}, a_{8}, a_{9}, b_{2}, b_{3}, b_{4}, b_{5}, b_{6}, b_{7}, b_{8}, b_{9}, c_{2}, c_{3}, c_{4}, c_{5}, c_{6}, c_{7}, \\
& c_{8}, \alpha_{1}, \alpha_{3}, \alpha_{4}, \alpha_{5}, \alpha_{6}, \alpha_{7}, \alpha_{8}, \alpha_{9}, \beta_{1}, \beta_{2}, \beta_{4}, \beta_{5}, \beta_{6}, \beta_{7}, \beta_{8}, \beta_{9}, \gamma_{1}, \gamma_{2}, \gamma_{3}, \gamma_{4}, \\
& \gamma_{5}, \gamma_{7}, \gamma_{8}, \gamma_{9} .
\end{aligned}
$$

The coefficient $C_{5}$ is given by

$$
\frac{\pi\left((a-2) a_{6}-a\left(2 \alpha_{6}+\alpha_{7}+a_{8}+2 a_{9}-\alpha_{9}\right)+2\left(\alpha_{7}+a_{8}-\alpha_{9}\right)\right)}{2 a^{3} \sqrt{(a-1) a^{2}}} .
$$

The coefficients $C_{1}, C_{2}$ and $C_{3}$ are mutually linearly independents. In fact, the parameter $\phi_{2}$ that appears in $C_{1}$ does not appear in $C_{3}$ and $C_{5}$ and the parameter $a_{1}$ that appears in $C_{3}$ does not appear in $C_{1}$ and $C_{5}$.

Therefore the limit cycles appear in the third, fourth and fifth order. The functions $f_{3}$ and $f_{4}$ have at most 1 simple zero and the function $f_{5}$ has at most 2 simple zeros and they are reached. This means that the perturbation of the class $P_{5}$ has at most 2 limit cycles by using the averaging theory of fifth order.

6.2. Proof for the Class $P_{5}$ with $d=0$. Take $d=0$ in the class $P_{5}$. The two centers obtained are symmetric with $x$-axis. So without loss of generality we consider the center $(-\beta,-\beta \gamma)$. Translating this center to the origin we obtain the system

$$
\begin{aligned}
& \dot{X}=P(X, Y)=(\beta-X)\left(-X\left(\gamma^{2}(X-2 \beta)+X\right)-2 \beta \gamma Y+Y^{2}\right) \\
& \dot{Y}=Q(X, Y)=(Y-\beta \gamma)\left(\left(\gamma^{2}+1\right) X(X-2 \beta)+2 \beta \gamma Y-Y^{2}\right)
\end{aligned}
$$

We perturb the previous system upto fifth order as follows

$$
\begin{aligned}
\dot{X} & =P(X, Y)+\varepsilon^{i} \sum_{i=1}^{5} p_{i}(X, Y), \\
\dot{Y} & =Q(X, Y)+\varepsilon^{i} \sum_{i=1}^{5} q_{i}(X, Y),
\end{aligned}
$$

where

$$
\begin{aligned}
p_{1}(X, Y)= & a_{1} X+a_{2} Y+a_{3} X^{2}+a_{4} X Y+a_{5} Y^{2}+a_{6} X^{3}+a_{7} X^{2} Y \\
& +a_{8} X Y^{2}+a_{9} Y^{3}, \\
q_{1}(X, Y)= & \alpha_{1} X+\alpha_{2} Y+\alpha_{3} X^{2}+\alpha_{4} X Y+\alpha_{5} Y^{2}+\alpha_{6} X^{3}+\alpha_{7} X^{2} Y \\
& +\alpha_{8} X Y^{2}+\alpha_{9} Y^{3} .
\end{aligned}
$$

The polynomials $p_{2}, p_{3}, p_{4}$ and $p_{5}$ are given changing the character $a$ by $b, c, d$ and $e$ in $p_{1}$ respectively and the polynomials $q_{2}, q_{3}, q_{4}$ and $q_{5}$ are obtained changing the character $\alpha$ by $\beta, \gamma, \delta$ and $\phi$ respectively. 
Now to study the bifurcations surround the origin we take the variable changes $X=\varepsilon x$ and $Y=\varepsilon y$ and we pass the system obtained in the variables $x$ and $y$ to the real Jordan Form. Due the size of the system obtained in the real Jordan form we do not give it explicitly here. After we pass the system to the polar coordinates taking $x=r \cos \theta$ and $y=r \sin \theta$ and we take the quotient $\dot{r} / \dot{\theta}$ and we obtain the differential equation in the standard form of the averaging of fifth order

$$
\frac{d r}{d \theta}=\sum_{i=1}^{5} K_{i} \varepsilon_{i} .
$$

The coefficients $K_{i}$ are very large and we do not explicitly it here. Calculating the functions $f_{i}$ as in Theorem 4 we obtain

$$
f_{1}=-\frac{\pi r\left(a_{1}+\alpha_{2}\right)}{2 \beta^{2} \gamma} \text {. }
$$

Take $\alpha_{2}=-a_{1}$. So we have $f_{1}=0$ and we obtain following the process in Theorem 4

$$
f_{2}=-\frac{\pi r\left(b_{1}+\beta_{2}\right)}{2 \beta^{2} \gamma}
$$

Take $\beta_{2}=-b_{1}$. Thus $f_{2}=0$ and we get

$$
\begin{aligned}
f_{3}= & \frac{\pi r^{3}}{8 \beta^{4} \gamma^{3}}\left(2 a_{1}-2 a_{2} \gamma^{3}-2 a_{3} \beta \gamma^{2}-2 a_{4} \beta \gamma^{3}-2 a_{5} \beta \gamma^{4}-2 a_{5} \beta \gamma^{2}-3 a_{6} \beta^{2} \gamma^{2}\right. \\
& -2 a_{7} \beta^{2} \gamma^{3}-a_{8} \beta^{2} \gamma^{4}-a_{8} \beta^{2} \gamma^{2}-3 \alpha_{9} \beta^{2} \gamma^{4}-2 \alpha_{8} \beta^{2} \gamma^{3}-\alpha_{7} \beta^{2} \gamma^{2} \\
& \left.-3 \alpha_{9} \beta^{2} \gamma^{2}-2 \alpha_{5} \beta \gamma^{3}-2 \alpha_{4} \beta \gamma^{2}-2 \alpha_{3} \beta \gamma-2 \alpha_{5} \beta \gamma-2 \alpha_{1} \gamma\right)-\frac{\pi r\left(c_{1}+\gamma_{2}\right)}{2 \beta^{2} \gamma} .
\end{aligned}
$$

Solving in the variable $a_{4}$ the equation obtained equalling to zero the factor that multiplies $\frac{\pi r^{3}}{8 \beta^{4} \gamma^{3}}$ and taking $c_{1}=-\gamma_{2}$ we have $f_{3}=0$. Following the process we obtain

$$
f_{4}=A_{1} r+A_{3} r^{3}
$$

where

$$
A_{1}=-\frac{\pi r\left(d_{1}+\delta_{2}\right)}{2 \beta^{2} \gamma}
$$

The coefficient $A_{3}$ is too large thus we do not explicitly it here but it is given in the variables

$$
\begin{aligned}
& \beta, \gamma, a_{1}, a_{3}, a_{5}, a_{6}, a_{8}, a_{2}, \alpha_{1}, a_{7}, \alpha_{3}, \alpha_{4}, \alpha_{5}, \alpha_{7}, \alpha_{8}, \alpha_{9}, b_{3}, b_{5}, b_{6}, b_{8}, b_{2}, b_{4}, \\
& \beta_{7}, \beta_{1}, \beta_{2}, \beta_{3}, \beta_{4}, \beta_{5}, \beta_{7}, \beta_{8}, \beta_{9} .
\end{aligned}
$$

The coefficients $A_{1}$ and $A_{3}$ are linearly independent because the parameter $d_{1}$ that appears in $A_{1}$ does not appear in $A_{3}$. Taking $d_{1}=-\delta_{2}$ and solving $A_{3}=0$ in the variable $\beta_{3}$ we have that $f_{4}=0$. Following the process we obtain

$$
f_{5}=C_{1} r+C_{3} r^{3}+C_{5} r^{5}
$$


where

$$
C_{1}=-\frac{\pi r\left(e_{1}+\phi_{2}\right)}{2 \beta^{2} \gamma}
$$

Again we do not have explicitly $C_{3}$ but it is given in the variables

$$
\begin{aligned}
& \beta, \gamma, a_{1}, a_{3}, a_{5}, a_{6}, a_{8}, a_{7}, a_{2}, \alpha_{1}, \alpha_{3}, \alpha_{4}, \alpha_{5}, \alpha_{7}, \alpha_{8}, \alpha_{9}, b_{2}, \beta_{1}, \beta_{2}, b_{3}, b_{5}, \\
& b_{4}, \beta_{4}, \beta_{5}, b_{6}, b_{8}, b_{7}, \beta_{7}, \beta_{8}, \beta_{9}, c_{2}, c_{3}, c_{4}, c_{5}, c_{6}, c_{7}, c_{8}, \gamma_{1}, \gamma_{2}, \gamma_{3}, \gamma_{4}, \gamma_{5}, \gamma_{7}, \\
& \gamma_{8}, \gamma_{9} .
\end{aligned}
$$

The coefficient $C_{5}$ is given by

$$
\frac{\pi\left(\gamma\left(-a_{6}+\alpha_{7}+a_{8} \gamma^{2}+a_{8}-\alpha_{9}\left(\gamma^{2}+1\right)\right)+2 \alpha_{6}+2 a_{9}\left(\gamma^{2}+1\right)^{2}\right)}{16 \beta^{4} \gamma^{2}} .
$$

The coefficients $C_{1}, C_{2}$ and $C_{3}$ are mutually linearly independents. In fact, the parameter $\phi_{2}$ that appears in $C_{1}$ does not appear in $C_{3}$ and $C_{5}$ and the parameter $a_{1}$ that appears in $C_{3}$ does not appear in $C_{1}$ and $C_{5}$.

Thus, the limit cycles appear in the third, fourth and fifth order. The functions $f_{3}$ and $f_{4}$ have at most 2 simple zeros and the function $f_{5}$ has at most 3 simple zeros and they are reached. This means that the perturbation of the class $P_{5}$ for $d=0$ has at most 2 limit cycles by using the averaging theory of fifth order.

We can repeat the process for $d=1$ with the same tools and conclude that the class $P_{5}$ has at most 2 limit cycles and they are reached.

\section{Example with 3 Cycles for the Class $P_{5}$}

Now we present an example with 3 limit cycles for the class $P_{5}$. This example was obtained for $d=0$. As in the previous subsection we perturb the system up to seventh order as follows

$$
\begin{aligned}
\dot{X} & =P(X, Y)+\varepsilon^{i} \sum_{i=1}^{7} p_{i}(X, Y), \\
\dot{Y} & =Q(X, Y)+\varepsilon^{i} \sum_{i=1}^{7} q_{i}(X, Y),
\end{aligned}
$$

where

$$
\begin{aligned}
p_{1}(X, Y)= & a_{1} X+a_{2} Y+a_{3} X^{2}+a_{4} X Y+a_{5} Y^{2}+a_{6} X^{3}+a_{7} X^{2} Y \\
& +a_{8} X Y^{2}+a_{9} Y^{3} \\
q_{1}(X, Y)= & \alpha_{1} X+\alpha_{2} Y+\alpha_{3} X^{2}+\alpha_{4} X Y+\alpha_{5} Y^{2}+\alpha_{6} X^{3}+\alpha_{7} X^{2} Y \\
& +\alpha_{8} X Y^{2}+\alpha_{9} Y^{3} .
\end{aligned}
$$

The polynomials $p_{2}, p_{3}, p_{4}, p_{5}, p_{6}$ and $p_{7}$, are given changing the character $a$ by $b, c, d, e, i$ and $j$ in $p_{1}$ respectively and the polynomials $q_{2}, q_{3}, q_{4}, q_{5}, q_{6}$ and $q_{7}$ are obtained changing the character $\alpha, \beta, \gamma, \delta, \phi, \iota$ and $\varphi$ respectively. 
We vanish some coefficients of the polynomials $p_{i}$ and $q_{i}$ for $i=1, \ldots, 7$. We vanish the coefficients

$$
\begin{aligned}
& a_{3}, a_{5}, a_{7}, a_{8}, a_{9}, b_{1}, b_{3}, b_{4}, b_{5}, b_{6}, b_{7}, b_{8}, c_{2}, c_{4}, c_{5}, c_{6}, c_{7}, c_{9}, d_{1}, d_{2}, d_{3}, d_{4}, d_{5}, \\
& d_{7}, d_{8}, d_{9}, e_{3}, e_{4}, e_{5}, e_{6}, e_{7}, e_{8}, e_{9}, i_{2}, i_{3}, i_{4}, i_{5}, i_{6}, i_{7}, i_{8}, i_{9}, j_{2}, j_{3}, j_{4}, j_{5}, j_{6}, j_{7}, \\
& j_{8}, j_{9}, \alpha_{1}, \alpha_{2}, \alpha_{3}, \alpha_{4}, \alpha_{5}, \alpha_{6}, \alpha_{8}, \beta_{1}, \beta_{4}, \beta_{5}, \beta_{6}, \beta_{7}, \beta_{8}, \gamma_{1}, \gamma_{3}, \gamma_{4}, \gamma_{5}, \gamma_{6}, \gamma_{7}, \\
& \gamma_{8}, \delta_{1}, \delta_{3}, \delta_{4}, \delta_{5}, \delta_{7}, \delta_{8}, \delta_{9}, \phi_{1}, \phi_{3}, \phi_{4}, \phi_{5}, \phi_{6}, \phi_{7}, \phi_{8}, \phi_{9}, \iota_{1}, \iota_{3}, \iota_{4}, \iota_{5}, \iota_{7}, \iota_{8}, \iota_{9}, \\
& \varphi_{1}, \varphi_{3}, \varphi_{4}, \varphi_{5}, \varphi_{6}, \varphi_{7}, \varphi_{8}, \varphi_{9} .
\end{aligned}
$$

As before, taking $X=\varepsilon x$ and $Y=\varepsilon y$, passing the system for the real Jordan Form and after for the polar coordinates we obtain

$$
\frac{d r}{d \theta}=\sum_{i=1}^{7} K_{i} \varepsilon_{i} .
$$

Calculating the functions $f_{i}$ as in Theorem 4 we have

$$
f_{1}=-\frac{\pi a_{1} r}{2 \beta^{2} \gamma}
$$

Take $a_{1}=0$. Thus $f_{1}=0$ and following the process we have

$$
f_{2}=-\frac{\pi \beta_{2} r}{2 \beta^{2} \gamma}
$$

Take $\beta_{2}=0$. So $f_{2}=0$ and we get

$$
\begin{aligned}
f_{3}= & \frac{\pi r^{3}\left(-2 a_{2} \gamma^{3}-2 a_{4} \beta \gamma^{3}-3 a_{6} \beta^{2} \gamma^{2}-3 \alpha_{9} \beta^{2} \gamma^{4}-\alpha_{7} \beta^{2} \gamma^{2}-3 \alpha_{9} \beta^{2} \gamma^{2}\right)}{8 \beta^{4} \gamma^{3}} \\
& -\frac{\pi r\left(c_{1}+\gamma_{2}\right)}{2 \beta^{2} \gamma}
\end{aligned}
$$

Take

$$
a_{4}=\frac{-2 \alpha-2 \gamma-3 a_{6} \beta^{2}-\alpha_{7} \beta^{2}-3 \alpha_{9} \beta^{2}-3 \alpha_{9} \beta^{2} \gamma^{2}}{2 \beta \gamma}, \quad c_{1}=-\gamma_{2} .
$$

Thus $f_{3}=0$ and we obtain

$$
f_{4}=D_{1} r+D_{3} r^{3}
$$

where

$$
\begin{aligned}
D_{1}= & -\frac{\pi \delta_{2}}{2 \beta^{2} \gamma}, \\
D_{3}= & -\frac{1}{64 \beta^{6} \gamma^{4}} \pi\left(2 a_{2}^{2}\left(\gamma^{3}+\gamma\right)+a_{2} \beta^{2}\left(\left(-2 \gamma^{4}-7 \gamma^{2}-1\right)\left(3 a_{6}+\alpha_{7}\right)\right.\right. \\
& \left.-3 a_{9}\left(\gamma^{2}+1\right)^{2}\left(2 \gamma^{2}+1\right)\right)+\beta^{2} \gamma\left(9 a_{6}^{2} \beta^{2}\left(\gamma^{2}+1\right)+6 a_{6} \beta^{2}\left(\gamma^{2}\right.\right. \\
& +1)\left(\alpha_{7}+3 \alpha_{9}\left(\gamma^{2}+1\right)\right)+\beta\left(\beta ( \gamma ^ { 2 } + 1 ) \left(\left(\alpha_{7}+3 \alpha_{9}\left(\gamma^{2}+1\right)\right)^{2}\right.\right. \\
& \left.\left.\left.\left.+24 \beta_{9} \gamma^{2}\right)+16 \beta_{3} \gamma\right)+16 b_{2} \gamma^{3}\right)\right) .
\end{aligned}
$$


Taking $\delta_{2}=0$ and solving $D_{3}$ in the variable $\beta_{3}$ we have that $f_{4}=0$. In sequel we obtain

$$
f_{5}=E_{1} r+E_{3} r^{3}+E_{5} r^{5}
$$

where

$$
E_{1}=-\frac{\pi\left(e_{1}+\phi_{2}\right)}{2 \beta^{2} \gamma}, \quad E_{5}=-\frac{\pi\left(a_{6}-\alpha_{7}+\alpha_{9}+\alpha_{9} \gamma^{2}\right)}{16 \beta^{4} \gamma} .
$$

Due the size of coefficient $E_{3}$ we do not give it explicitly here but it is given in the variables

$$
\beta, \gamma, a_{2}, a_{6}, \alpha_{7}, \alpha_{9}, b_{2}, \beta_{9}, \gamma_{2}, c_{8}, \gamma_{9}
$$

Taking $e_{1}=-\phi_{2}, \alpha_{9}=\frac{\alpha_{7}-a_{6}}{1+\gamma^{2}}$ and solving $E_{3}=0$ in the variable $b_{2}$ we have $f_{5}=0$. Following the process we have

$$
f_{6}=F_{1} r+F_{3} r^{3}+F_{5} r^{5}
$$

where

$$
F_{1}=-\frac{\pi\left(i_{1}+\iota_{2}\right)}{2 \beta^{2} \gamma} .
$$

Again we do not give the coefficients $F_{3}$ and $F_{5}$ here but $F_{3}$ is given in the variables

$$
\beta, \gamma, a_{2}, \alpha_{7}, \alpha_{6}, b_{2}, \beta_{9}, d_{6}, \gamma_{2}, c_{8}, \gamma_{9}
$$

and $F_{5}$ is given in the variables

$$
\beta, \gamma, a_{2}, a_{6}, \alpha_{7}, b_{9}, \beta_{9} .
$$

Taking $i_{1}=-\iota_{2}$ and solving $F_{3}=0$ and $F_{5}=0$ respectively in the variables $d_{6}$ and $b_{9}$ we have $f_{6}=0$. Finally we have

$$
f_{7}=G_{1} r+G_{2} r^{2}+G_{3} r^{3}+G_{5} r^{5}
$$

where

$$
G_{2}=\frac{\pi\left(a_{2}\left(\gamma^{2}+5\right) \phi_{2}-16 \beta^{2} \gamma\left(2 \alpha_{7} \phi_{2}+3 \iota_{2}\right)\right)}{192 \beta^{5} \gamma^{3}} .
$$

The remain coefficients are too large and we do not give they here. However the coefficient $G_{1}$ is given in the variables

$$
\beta, \gamma, a_{2}, \alpha_{7}, a_{6}, \phi_{2}, \iota_{2}, \beta_{9}, \varphi_{2}, \gamma_{2}, c_{8}, \gamma_{9},
$$

the coefficient $G_{2}$ is given in the variables

$$
\beta, \gamma, a_{2}, \phi_{2}, \iota_{2}, \alpha_{7},
$$

the coefficient $G_{3}$ is given in the variables

$$
\beta, \gamma, a_{2}, \alpha_{7}, a_{6}, \beta_{9}, \gamma_{2}, c_{8}, \gamma_{9}, e_{2}, \phi_{2},
$$

and the coefficient $G_{5}$ is given in the variables

$$
\beta, \gamma, a_{2}, \alpha_{7}, a_{6}, \beta_{9}, \gamma_{2}, c_{8}, \gamma_{9} .
$$

We can note that the coefficients $G_{1}, G_{2}, G_{3}$ and $G_{5}$ are linearly independents. So the function $f_{7}$ has at most 3 simple zeros and they are reached. These zeros correspond to limit cycles for the class $P_{5}$. 


\section{Appendix A. Computations of the FunCtions $y_{6}$ And $y_{7}$}

Now we give explicitly the functions $y_{6}$ and $y_{7}$ stated in Theorem 4 which has been used to obtain an example with 3 limit cycles for the class $P_{5}$ in Section 7 .

The functions $y_{6}$ and $y_{7}$ are given respectively by

$$
\begin{aligned}
& y_{6}(t, z)= \int_{0}^{t}\left(720 F_{6}(s, z)+720 \frac{\partial F_{5}}{\partial x}(s, z) y_{1}(s, z)+360 \frac{\partial^{2} F_{4}}{\partial x^{2}}(s, z) y_{1}(s, z)^{2}\right. \\
&+ 360 \frac{\partial F_{4}}{\partial x}(s, z) y_{2}(s, z)+360 \frac{\partial^{2} F_{3}}{\partial x^{2}}(s, z) y_{1}(s, z) \odot y_{2}(s, z) \\
&+120 \frac{\partial^{3} F_{3}}{\partial x^{3}}(s, z) y_{1}(s, z)^{3}+120 \frac{\partial F_{3}}{\partial x}(s, z) y_{3}(s, z) \\
&+120 \frac{\partial^{2} F_{2}}{\partial x^{2}}(s, z) y_{1}(s, z) \odot y_{3}(s, z) \\
&+ 90 \frac{\partial^{2} F_{2}}{\partial x^{2}}(s, z) y_{2}(s, z)^{2}+180 \frac{\partial^{3} F_{2}}{\partial x^{3}}(s, z) y_{1}(s, z)^{2} \odot y_{2}(s, z) \\
&+30 \frac{\partial^{4} F_{2}}{\partial x^{4}}(s, z) y_{1}(s, z)^{4}+30 \frac{\partial F_{2}}{\partial x}(s, z) y_{4}(s, z) \\
&+ 30 \frac{\partial^{2} F_{1}}{\partial x^{2}}(s, z) y_{1}(s, z) \odot y_{4}(s, z)+60 \frac{\partial^{3} F_{1}}{\partial x^{3}}(s, z) y_{1}(s, z)^{2} \odot y_{3}(s, z) \\
&+ 60 \frac{\partial^{4} F_{1}}{\partial x^{4}}(s, z) y_{1}(s, z)^{3} \odot y_{2}(s, z)+90 \frac{\partial^{3} F_{1}}{\partial x^{3}}(s, z) y_{2}(s, z)^{2} \odot y_{1}(s, z) \\
&+ 6 \frac{\partial^{5} F_{1}}{\partial x^{5}}(s, z) y_{1}(s, z)^{5}+60 \frac{\partial^{2} F_{1}}{\partial x^{2}}(s, z) y_{2}(s, z) \odot y_{3}(s, z) \\
&+\left.6 \frac{\partial F_{1}}{\partial x}(s, z) y_{5}(s, z)\right) d s, \\
&+210 \frac{\partial^{2} F_{2}}{\partial x^{2}}(s, z) y_{1}(s, z) \odot y_{4}(s, z) \\
&+630 \frac{\partial^{2} F_{3}}{\partial x^{2}}(s, z) y_{2}(s, z)^{2}+1260 \frac{\partial^{3} F_{3}}{\partial x^{3}}(s, z) y_{1}(s, z)^{2} \odot y_{2}(s, z) \\
&+210 \frac{\partial^{4} F_{3}}{\partial x^{4}}(s, z) y_{1}(s, z)^{4}+210 \frac{\partial F_{3}}{\partial x}(s, z) y_{4}(s, z) \\
&+2520 \frac{\partial^{2} F_{5}}{\partial x^{2}}(s, z) y_{1}(s, z)^{2}+2520 \frac{\partial F_{5}}{\partial x}(s, z) y_{2}(s, z) \\
&+ 2520 \frac{\partial^{2} F_{4}}{\partial x^{2}}(s, z) y_{1}(s, z) \odot y_{2}(s, z)+840 \frac{\partial^{3} F_{4}}{\partial x^{3}}(s, z) y_{1}(s, z)^{3} \\
& y_{7}(t, z)(s, z) y_{3}(s, z)+840 \frac{\partial^{2} F_{3}}{\partial x^{2}}(s, z) y_{1}(s, z) \odot y_{3}(s, z) \\
&=
\end{aligned}
$$




$$
\begin{aligned}
& +420 \frac{\partial^{3} F_{2}}{\partial x^{3}}(s, z) y_{1}(s, z)^{2} \odot y_{3}(s, z) \\
& +420 \frac{\partial^{4} F_{2}}{\partial x^{4}}(s, z) y_{1}(s, z)^{3} \odot y_{2}(s, z) \\
& +630 \frac{\partial^{3} F_{2}}{\partial x^{3}}(s, z) y_{2}(s, z)^{2} \odot y_{1}(s, z)+42 \frac{\partial^{5} F_{2}}{\partial x^{5}}(s, z) y_{1}(s, z)^{5} \\
& +420 \frac{\partial^{2} F_{2}}{\partial x^{2}}(s, z) y_{2}(s, z) \odot y_{3}(s, z)+42 \frac{\partial F_{2}}{\partial x}(s, z) y_{5}(s, z) \\
& +630 \frac{\partial^{3} F_{2}}{\partial x^{3}}(s, z) y_{2}(s, z)^{2} \odot y_{1}(s, z)+7 \frac{\partial^{6} F_{1}}{\partial x^{6}}(s, z) y_{1}(s, z)^{6} \\
& +105 \frac{\partial^{5} F_{1}}{\partial x^{5}}(s, z) y_{1}(s, z)^{4} \odot y_{2}(s, z) \\
& +140 \frac{\partial^{4} F_{1}}{\partial x^{4}}(s, z) y_{1}(s, z)^{3} \odot y_{3}(s, z) \\
& +630 \frac{\partial^{4} F_{1}}{\partial x^{4}}(s, z) y_{1}(s, z)^{2} \odot y_{2}(s, z)^{2} \\
& +105 \frac{\partial^{3} F_{1}}{\partial x^{3}}(s, z) y_{1}(s, z)^{2} \odot y_{4}(s, z) \\
& +42 \frac{\partial^{2} F_{1}}{\partial x^{2}}(s, z) y_{1}(s, z) \odot y_{5}(s, z) \\
& +420 \frac{\partial^{3} F_{1}}{\partial x^{3}}(s, z) y_{1}(s, z) \odot y_{2}(s, z) \odot y_{3}(s, z) \\
& +105 \frac{\partial^{3} F_{1}}{\partial x^{3}}(s, z) y_{2}(s, z)^{3}+105 \frac{\partial^{2} F_{1}}{\partial x^{2}}(s, z) y_{2}(s, z) \odot y_{4}(s, z) \\
& \left.+70 \frac{\partial^{2} F_{1}}{\partial x^{2}}(s, z) y_{3}(s, z)^{2}+7 \frac{\partial F_{1}}{\partial x}(s, z) y_{6}(s, z)\right) d s .
\end{aligned}
$$

\section{ACKNOWLEDGEMEnTS}

We thank to the reviewers their comments and suggestions which help us to improve this paper.

The first author is partially supported by a MINECO/FEDER grant MTM2008-03437 and MTM2013-40998-P, an AGAUR grant number 2014 SGR568, an ICREA Academia, the grants FP7-PEOPLE-2012-IRSES 318999 and 316338, FEDER-UNAB-10-4E-378, and a CAPES grant number 88881. 030454/2013-01 from the program CSF-PVE. The second authors is partially supported by the joint projects FP7-PEOPLE-2012-IRSES numbers 316338, CAPES grant number 88881.030454/2013-01 from the program CSF-PVE and CNPq grant "Projeto Universal 472796/2013-5". The second author is supported by CAPES/GDU - 7500/13-0. The last author is supported by FAPESP-2010/17956-1. 


\section{REFERENCES}

[1] A. A. Andronov, E. A. Leontocvich, I. I. Gordon and A. G. Maüer, Theory of bifurcations of dynamic systems on a plane, Halsted Press [A division of John Wiley \& Sons], New York-Toronto, Ont., 1973, Translated from the Russian.

[2] T. R. Blows and L. M. Perko, Bifurcation of limit cycles from centers and separatrix cycles of planar analytic systems, SIAM Rev. 36 (1994), 341-376.

[3] A. Buică and J. Llibre, Averaging methods for finding periodic orbits via Brower degree, Bull. Sci. Math. 128 (2004), no. 1, 7-22.

[4] C. Christopher and C. Li, Limit cycles in differential equations, Birkhauser, Boston, 1999.

[5] H. Giacomini, J. Llibre and M. Viano, On the shape of limit cycles that bifurcate from Hamiltonian centers, Nonlinear Anal. 41 (2000), 523-537.

[6] J. Giné, Higher order limit cycle bifurcations from non-degenerate centers, Appl. Math. Comput. 218 (2012), no. 17, 8853-8860.

[7] M. Han, Asymptotic expansions of Melnikov functions and limit cycle bifurcations, Int. J. Bifur. Chaos 22 (2012), no. 12, 1250296, 30 pp.

[8] M. Han, J. Yang and P. Yu, Hopf bifurcations for near-Hamiltonian systems, Int. J. Bifur. Chaos 19 (2009), 4117-4130.

[9] M. Han and P. Yu, Normal forms, Melnikov functions and bifurcations of limit cycles, vol. 181, Applied Mathematical Sciences, Springer, London 2012.

[10] C. Hilbert, Mathematische Probleme, Lecture, Second Internat. Congr. Math. (Paris, 1900), Nachr. Ges. Wiss. G"ttingen Math. Phys. KL. (1900), 253-297; English transl., Bull. Amer. Math. Soc. 8 (1902), 437-479; Bull. (New Series) Amer. Math. Soc. 37 (2000), 407-436.

[11] S. Karlin and W. J. Studden, T-systems: With applications in analysis and statistics, Pure Appl. Math., Interscience Publishers, New York, London, Sidney, 1966.

[12] C. Li, W. Li, J. Llibre and Z. Zhang, Linear estimation of the number of zeros of abelian integrals for some cubic isochronous centers, J. Differential Equations 180 (2002), 307-333.

[13] M. F. S. Lima and J. Llibre, Limit cycles and invariant cylinders for a class of continuous and discontinuous vector field in dimension 2n, Appl. Math. Comput. 217 (2011), no. 24, 9985-9996.

[14] J. Llibre, A. Mahdi and N. Vulpe, Phase portraits and invariant straight lines of cubic polynomial vector fields having a quadratic rational first integral, Rocky Mountain J. of Math. 41 (2011), 1585-1629.

[15] J. Llibre, D. D. Novaes and M. A. Teixeira, Higher order averaging theory for finding periodic solutions via Brouwer degree, Nonlinearity 27 (2014), 563-583.

[16] S. Smale, Mathematical problems for next century, Math. Intelligencer 20 (1998), no. $2,7-15$.

[17] L. Yang, Recent Advances on Determining the Number of Real Roots of Parametric Polynomials, J. Symbolic Computation 28 (1999), 225-242.

[18] Y. Yanqian, Theory of Limit Cycles, vol. 66, Transl. Math. Monogr., American Mathematical Society, Providence, RI, 1986.

[19] W. Yusen and C. Zang, Bifurcation of limit cycles and pseudo-isochronous center at degenerate singular point in a septic system, Appl. Math. Comput. 218 (2012), no. $17,8513-8525$.

\footnotetext{
${ }^{1}$ Departament de Matemàtiques, Universitat Autònoma de Barcelona, 08193 Bellaterra, Barcelona, Catalonia, Spain.

E-mail address: jllibre@mat.uab.cat

E-mail address: blopes@mat.uab.cat
} 
2 Departamento de Matemática - IBIlCe-Unesp, Rua C. Colombo, 2265, CEP 15054-000 S. J. Rio Preto, SÃo Paulo, Brazil.

E-mail address: jrezmoraes@gmail.com 